\title{
Elliptical instability in terrestrial planets and moons
}

\author{
D. Cebron ${ }^{1}$, M. Le Bars ${ }^{1}$, C. Moutou ${ }^{2}$, and P. Le Gal ${ }^{1}$ \\ 1 Institut de Recherche sur les Phénomènes Hors Equilibre, UMR 6594, CNRS and Aix-Marseille Université, 49 rue F. Joliot-Curie, \\ BP 146, 13384 Marseille Cedex 13, France \\ e-mail: cebron@irphe.univ-mrs.fr \\ 2 Observatoire Astronomique de Marseille-Provence, Laboratoire d'Astrophysique de Marseille, 38 rue F. Joliot-Curie, \\ 13388 Marseille Cedex 13, France
}

Received 21 July 2011 / Accepted 16 January 2012

\section{ABSTRACT}

\begin{abstract}
Context. The presence of celestial companions means that any planet may be subject to three kinds of harmonic mechanical forcing: tides, precession/nutation, and libration. These forcings can generate flows in internal fluid layers, such as fluid cores and subsurface oceans, whose dynamics then significantly differ from solid body rotation. In particular, tides in non-synchronized bodies and libration in synchronized ones are known to be capable of exciting the so-called elliptical instability, i.e. a generic instability corresponding to the destabilization of two-dimensional flows with elliptical streamlines, leading to three-dimensional turbulence.

Aims. We aim here at confirming the relevance of such an elliptical instability in terrestrial bodies by determining its growth rate, as well as its consequences on energy dissipation, on magnetic field induction, and on heat flux fluctuations on planetary scales.

Methods. Previous studies and theoretical results for the elliptical instability are re-evaluated and extended to cope with an astrophysical context. In particular, generic analytical expressions of the elliptical instability growth rate are obtained using a local WKB approach, simultaneously considering for the first time (i) a local temperature gradient due to an imposed temperature contrast across the considered layer or to the presence of a volumic heat source and (ii) an imposed magnetic field along the rotation axis, coming from an external source.

Results. The theoretical results are applied to the telluric planets and moons of the solar system as well as to three Super-Earths: $55 \mathrm{CnC}$ e, CoRoT-7b, and GJ 1214b. For the tide-driven elliptical instability in non-synchronized bodies, only the early Earth core is shown to be clearly unstable. For the libration-driven elliptical instability in synchronized bodies, the core of Io is shown to be stable, contrary to previously thoughts, whereas Europa, $55 \mathrm{CnC}$ e, CoRoT-7b, and GJ 1214b cores can be unstable. The subsurface ocean of Europa is slightly unstable. However, these present states do not preclude more unstable situations in the past.
\end{abstract}

Key words. hydrodynamics - instabilities - planets and satellites: interiors - planets and satellites: dynamical evolution and stability

\section{Introduction}

The flows in fluid layers of planets and moons are of major interest because they imply first-order consequences for their internal dynamics and orbital evolutions. Indeed, internal flows create torques on solid layers and induce energy dissipation. Moreover, internal flows are directly responsible for the generation of magnetic fields, either by induction of an existing background magnetic field or by excitation of a self-sustained dynamo. Finally, planetary heat fluxes are also directly linked to flows in fluid layers, which can act as thermal blankets for stably stratified configurations, or as efficient heat flux conveyers in the case of convective flows.

Planetary fluid layers are subject to body rotation, which implies that inertial waves can propagate through them (e.g. Greenspan 1968). Usually damped by viscosity, these waves can, however, be excited by longitudinal libration, precession, and tides, which are harmonic mechanical forcings of azimuthal periodicity $m=0,1$, and 2 , respectively. The fluid response to mechanical forcings in ellipsoids is a long-standing issue: see e.g. for longitudinal libration Aldridge \& Toomre (1969), Noir et al. (2009), Calkins et al. (2010), Sauret et al. (2010), Chan et al. (2011a) and Zhang et al. (2011), for latitudinal libration, Chan et al. (2011b), for precession, Poincaré (1910), Busse (1968), Cébron et al. (2010b) and for tides, Ogilvie \& Lin (2004), Ogilvie \& Lin (2007), Tilgner (2007), Rieutord \& Valdettaro (2010) and Morize et al. (2010). In these studies, it has been shown that the dynamics of a fluid layer is completely modified when the forcing resonates with an inertial wave. In addition to these direct forcings, inertial waves can also form triadic resonances, leading to parametric inertial instabilities. For instance, the so-called shear instability can be excited by precession (Kerswell 1993b; Lorenzani \& Tilgner 2001, 2003), and the elliptical instability can be excited by tides in non-synchronized bodies (Malkus 1989; Rieutord 2000) and by librations in synchronized ones (Kerswell \& Malkus 1998).

The elliptical instability is a generic instability that affects any rotating fluid whose streamlines are elliptically deformed (see the review by Kerswell 2002). A fully three-dimensional turbulent flow is excited in the bulk as soon as (i) the ratio between the ellipticity of the streamlines $\beta$ and the square root of the Ekman number $E$ (which represents the ratio between the viscous over the Coriolis forces) is more than a critical value on the order of one and (ii) as soon as a difference in angular velocity exists between the mean rotation rate of the fluid and the elliptical distortion. In a planetary context, the ellipticity of streamlines is related to the gravitational deformation of all layers of the considered body, coming from the static and periodic terms of the tidal potential, as well as from a potential frozen bulge. The differential rotation between the fluid and the elliptical distortion can be oscillatory when caused by libration in synchronized systems, or stationary in non-synchronized ones. The elliptical instability is then respectively referred to as 


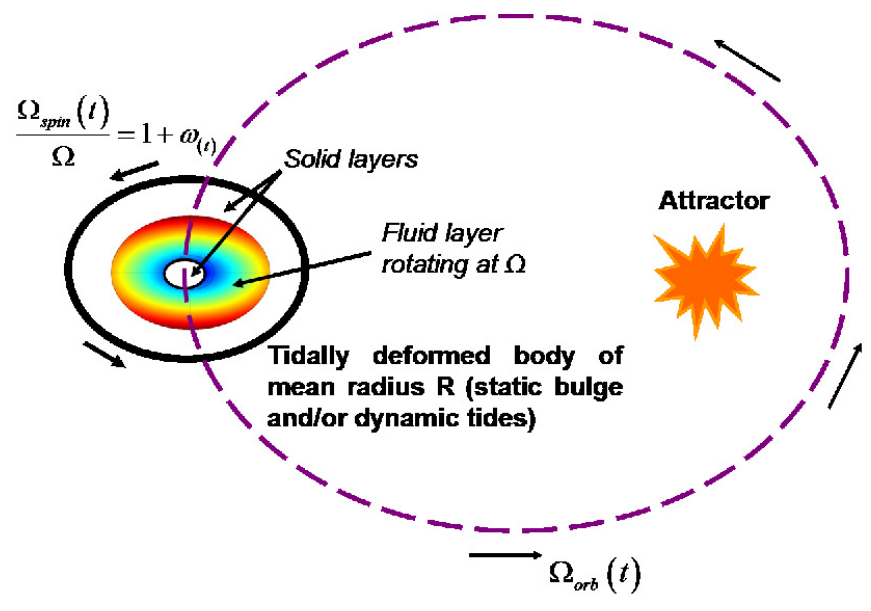

(a)

Fig. 1. Sketch of the problem studied in this work. With the mean rotation rate of the fluid $\Omega$, we define the dimensionless orbital rotation rate $\gamma_{(t)}$ and the dimensionless spin rotation rate $1+\omega_{(t)}$. The phase lag between the tide and the gravitational potential of the host body is not relevant to our purpose.

libration-driven elliptical instability (LDEI) and tide-driven elliptical instability (TDEI). TDEI and LDEI have already been suggested as taking place respectively on Earth (e.g. Aldridge et al. 1997) and on Io (e.g. Kerswell \& Malkus 1998). However, these previous works do not consider some planetary particularities, so they need to be revisited. For instance, Aldridge et al. (1997) did not take the orbital rate of the Moon into account or the magnetic field of the Earth, thus neglecting the effects of tide rotation and Joule dissipation on the growth of TDEI. Kerswell \& Malkus (1998) implicitly assumed that the tidal response of Io is completely fluid, neglecting the rigidity of its mantle and overestimating the amplitude of librations and tidal deformations. Our purpose here is to extend previous results of the literature on TDEI and LDEI and to determine general formulas for quantifying the presence of the elliptical instability in terrestrial bodies, taking the relevant complexities present in natural systems into account.

This paper is organized as follows. Section 2 presents the different celestial forcings that could excite an elliptical instability, first focusing on tides in non-synchronized systems, and then on forced and free libration in synchronized ones. In Sect. 3, we introduce our physical model and develop a local WKB analysis in all configurations, including the effects of viscosity, as well as the effects of an imposed magnetic field and a local temperature gradient. These theoretical results are used in Sect. 4 to investigate the possible presence of any of TDEI and LDEI in telluric planets and moons of the solar system, as well as in two Super-Earths of extrasolar systems. The possible consequences of those instabilities are finally considered.

\section{From celestial mechanics to the excitation of an elliptical instability}

Figure 1 presents a sketch of the problem considered in this work. We consider a telluric body of rotation rate $\Omega_{\text {spin }}$, orbiting around an attractor (orbit in black dashed line) at the orbital rate $\Omega_{\text {orb }}$. This body has a radius $R$, a mass $M$, and a fluid layer in its interior between an external radius $R_{2}$ and an internal radius $R_{1}$, typically a liquid outer core. We suppose that this internal fluid layer is enclosed between an external elliptically deformed
Table 1. List of the different astrophysical configurations that could lead to an elliptical instability (E.I) in a planetary fluid layer (liquid core, subsurface ocean) of a non synchronized or a synchronized celestial body.

\begin{tabular}{lcccc}
\hline \hline State & $\begin{array}{c}\text { Origin } \\
\text { of } \Delta \Omega\end{array}$ & $\begin{array}{c}\text { Origin } \\
\text { of } \beta\end{array}$ & $\Delta \Omega$ & E.I \\
\hline Non-sync. & spin rotation & D.T. & & \\
Non-sync. & impact & S.B. $^{a}$ & $\Omega_{\text {spin }}-\Omega_{\text {orb }}$ & TDin-up process \\
Sync. & forced O.L ${ }^{b}$ & D.T. & $2 e \Omega \cos \left(2 \pi t / T_{\text {orb }}\right)$ & TDEI \\
Sync. & forced P.L & LDI \\
Sync. & free P.L & S.B. & $\epsilon \Omega \cos \left(2 \pi t / T_{\text {orb }}\right)$ & LDEI \\
Sync. & any P.L & S.B., D.T. & $\epsilon \Omega \cos \left(\omega_{\text {free }} t\right)$ & LDEI \\
\hline
\end{tabular}

Notes. $\beta$ is the ellipticity of the boundaries distortion, $\Delta \Omega$ is the differential rotation rate between the fluid and the elliptical deformation, $\Omega$ is the mean spin rate of the planet, $\epsilon$ is the physical libration amplitude, $T_{\text {orb }}$ and $e$ are the orbital period and eccentricity, and $\omega_{\text {free }}$ is the free libration frequency. ${ }^{(a)}$ D.T. stands for dynamic tides and S.B. stands for static bulge. ${ }^{(b)}$ O.L. and P.L. stand respectively for optical and physical libration. ${ }^{(c)}$ Case equivalent to a non-synchronized case (cf. Sect. 2.2.2).

solid layer and a possible inner core, such as the outer liquid core of the Earth or the subsurface ocean of Europa. The elliptical deformation can admit different origins. First, in the presence of an orbiting companion, the elliptical deformation can come from the static and periodic terms of the tidal potential as seen from the mantle frame of reference. In this case, periodic terms lead to tides, and the static term leads to the so-called static (tidal) bulge (the solid layer behaves as a fluid layer on the long term). Second, a so-called frozen bulge, resulting from previous states, may exist, as for instance in the Moon (Garrick-Bethell et al. 2006). In this case, the body is not in hydrostatic equilibrium. For our purpose, it is sufficient to distinguish between the response to tidal potential periodic terms, which we call the dynamic tides, and a permanent (or very slowly changing) bulge, either owing to the tidal potential static terms or due to a frozen bulge, which we call a static bulge. The usual phase lag between the tide and the gravitational potential of the host body, which is due to internal dissipation, is not relevant for our purpose so is forgotten. Three dimensionless numbers are needed to describe the system: (i) the ellipticity $\beta$ of the elliptical deformation, (ii) the Ekman number $E=v /\left(\Omega R_{2}^{2}\right)$, where $v$ is the fluid kinematic viscosity, $R_{2}$ the outer radius of the rotating fluid, and $\Omega$ its typical angular velocity before any instability, equal to the mean value of the (possibly varying) mantle spin rate $\Omega_{\text {spin }}(t)$, (iii) the differential rotation $\Delta \Omega$ between the fluid and the elliptical distortion, non-dimensionalized by the fluid rotation rate, $\Delta \Omega / \Omega$. We distinguish two cases: a non-synchronized body, and a synchronized body. In the former, over one spin period, a mean differential rotation exists between the elliptical deformation and the fluid, whereas in the latter the mean rotation rates of the deformation and of the fluid are equal. The different cases are described in the following and summarized in Table 1.

\subsection{Non-synchronized bodies}

For a non-synchronized body, we consider two cases depending on the origin of the elliptical shape. First, if the spin rate of the mantle $\Omega_{\text {spin }}$ is constant, a TDEI can be excited by the tidal elliptical distortion due to dynamic tides, which rotate at the orbital velocity $\Omega_{\text {orb }}$. This is the standard configuration considered for instance by Craik (1989), who showed that TDEI is indeed 
possible except in a forbidden zone $\Omega_{\text {spin }} / \Omega_{\text {orb }} \in[-1 ; 1 / 3]$, where no triadic resonance is possible.

Second, if the elliptical shape comes from a static bulge, a non-zero mean differential rotation over one spin period implies that the fluid does not rotate at the same rate as the mantle, which corresponds to a spin-up or a spin-down process. This can occur, for instance, transiently after a large meteoritic impact, which is capable of fully desynchronizing the body (see for instance the considered scenario for explaining the Moon's magnetic field by Bars et al. 2011). In this case, a differential rotation exists between the fluid and the mantle with its static bulge, up to the typical spin-up/spin-down time necessary for the fluid to recover the mantle velocity (Greenspan 1968), i.e. up to

$t_{\text {spin-up }}=\Omega_{\text {spin }}^{-1} E^{-1 / 2}$.

Then, if the growth time of the TDEI is short enough compared to the spin-up time, one can expect a quasi-static evolution of the system, where the modification of the spin rate of the fluid is neglected during the growth of the instability: the former configuration is then transiently recovered.

\subsection{Synchronized bodies}

In the synchronized case, even if there is no mean differential rotation between the elliptical deformation and the fluid, oscillations can nevertheless occur for different reasons. For the study of the elliptical instability, it is necessary to know the amplitude of these oscillations, which depends on their origins. We distinguish below the forced librations caused by gravitational interactions with other celestial bodies, and free librations induced for instance by a meteoritic impact.

\subsubsection{Forced librations}

In forced librations, static bulge and dynamic tides have to be considered simultaneously. To illustrate this, following Goldreich \& Mitchell (2010), we consider a simple toy model without any internal dissipation: a synchronously spinning satellite, with an elastic outer shell and a homogeneous fluid interior, moving on an elliptic orbit. The orbital velocity changes along the orbit, and writes at first order in the orbital eccentricity $e$ as

$\Omega_{\text {orb }}=\Omega(1+2 e \cos \Omega t)$,

where $\Omega$ is the mean value of the mantle spin rate $\Omega_{\text {spin }}(t)$. Considering the influence of the orbital velocity variations on the satellite dynamics, we expect two limit cases: (i) if the rigidity of the elastic shell is zero or if the planet spin rate is low enough for the shape of the planet to have time to adapt to the gravitational constraints, the shell slides over the fluid and maintains its equilibrium shape, with the long axis of the ellipsoidal figure pointing toward the companion body; (ii) if the rigidity of the elastic shell is strong enough or if the planet spin rate is rather high, the entire satellite rotates rigidly with a fixed shape.

In the first case, only the elastic energy $E_{\text {elas }}$ varies: the meridians of the shell are stretched and compressed due to the rotation, whereas the spin velocity of the satellite remains constant. This is the so-called optical libration. In the second case, only the gravitational energy $E_{\text {grav }}$ varies and the spin velocity of the satellite changes, which corresponds to the so-called physical libration.

In both cases, the libration period remains small compared to the typical spin-up/spin-down time (1), which means that the fluid does not follows the solid boundaries because it never has enough time to adapt to the periodic velocity fluctuations and continues to rotate at the constant synchronous rotation rate $\Omega$. This is the so-called no spin-up condition. In the first case, therefore, a differential rotation exists between the fluid rotating at the constant rate $\Omega$ and the dynamic tides rotating at the oscillating orbital velocity, $\Delta \Omega / \Omega=2 e \cos \Omega t$. An LDEI can thus be excited by this optical libration, as shown theoretically by Kerswell \& Malkus (1998) and Herreman et al. (2009). In the second, a differential rotation $\Delta \Omega / \Omega=\epsilon \cos \Omega t$ exists between the fluid rotating at the constant rate $\Omega$ and the static bulge subject to physical librations of amplitude $\epsilon$, which depends on the internal structure of the satellite. The amplitude of the physical librations $\epsilon$ is always less than the $2 e$ extreme value given by optical libration, because of different internal torques such as the gravitational torque and elastic strain torque (see for instance Van Hoolst et al. 2008, 2009).

With the more general case of an arbitrary torque applied to the shell, Goldreich $\&$ Mitchell (2010) estimated the ratio $\mathfrak{Z}=$ $E_{\text {elas }} / E_{\text {grav }}$ by

$\mathfrak{R}=\frac{32 \pi}{5} \frac{1+\tilde{v}}{5+\tilde{v}} \frac{\left(1+k_{\mathrm{f}}\right)^{2}}{k_{\mathrm{f}}} \frac{\tilde{\mu} d R^{3}}{G M^{2}}$

where $\tilde{v}$ is the Poisson ratio, $k_{\mathrm{f}}$ the fluid Love number, $\tilde{\mu}$ the shell rigidity, $R$ and $d$ the mean radius and the thickness of the shell, $M$ the mass of the satellite. According to Goldreich \& Mitchell (2010), typical values give $\Re \sim 10^{-2}$ for the subsurface ocean of Europa, and $\mathfrak{R} \sim 0.1$ for the subsurface ocean of Titan, whereas the silicate mantle of Io is expected to behave in the limit $\mathfrak{R} \gg 1$. Because of the visco-elastic rheology of real bodies, the effective response should be between these two extrem cases given by this model. Goldreich \& Mitchell (2010) argue that the total increase in energy would be minimal, which leads us to consider that Europa and Titan, for instance, behave like entirely fluid satellites. In contrast, Karatekin et al. (2008), Van Hoolst et al. (2008, 2009), and Baland \& Van Hoolst (2010) consider that the arguments proposed by Goldreich \& Mitchell (2010) are unrealistic on short timescales, hence that the rheology does not allow the bodies to reach their minimal energetic state. They assume that they behave rigidly, however, large libration may still be due to resonances with free libration modes, which may be reached for thin ice shells (Baland \& Van Hoolst 2010). In addition to the displacements of the ice shell and mantle induced by gravitational interactions, relatively large longitudinal displacements may also be induced directly in the fluid layers by the periodical part of the tidal potential (Tobie et al. 2005). This would constitute a supplementary origin for a non-zero differential rotation between the fluid and the deformation. Either way, all these issues are still being debated and are clearly beyond the scope of the present paper. All that is needed here is to know the amplitude of the relative motion between the elliptical distortion and the fluid. In the following, we consider the full range of configurations up to a maximum distortion amplitude given by the extreme value of optical libration.

\subsubsection{Free librations}

After a meteoritic impact, for instance, so-called free librations can occur on the typical resynchronization time (e.g. Williams et al. 2001). Following the no spin-up condition explained in Sect. 2.2.1, the fluid keeps rotating at the orbital velocity (synchronized state), while the mantle librates around this mean value. The amplitude $\epsilon$ of the free librations depends initially on the impact strength and decreases through time, and the libration 
frequency remains equal to a proper frequency of the body, given by $\omega_{\text {free }}=\Omega \sqrt{3(B-A) / C}$ at first order in the orbital eccentricity, where $(A, B, C)$ are the three principal moments of inertia of the body (see for instance Lissauer 1985). Considering a static bulge, free librations can thus drive an LDEI from the differential rotation $\Delta \Omega / \Omega=\epsilon \cos \left(\omega_{\text {free }} t\right)$, providing that the growth time of the instability is shorter than the resynchronization time, as shown in Sect. 3.5.

\subsubsection{Zonal wind induced by physical librations}

Finally, in all scenarios involving physical librations, it has recently been determined analytically by Busse (2010), and confirmed experimentally and numerically by Sauret et al. (2010), that non-linearities in the Ekman layer driven by the librating rigid boundaries induce a differential rotation in the fluid of amplitude $\Delta \Omega / \Omega=-0.154\left(\theta \omega_{0} / \Omega\right)^{2}$, where $\theta$ is the amplitude angle of the libration and $\omega_{\mathrm{o}}$ its frequency. TDEI can thus be excited by this differential rotation with both static bulge and dynamic tides. Nevertheless, the differential rotation generated by this process is always very small. We do not expect this mechanism to play an important role in a planetary context, since it is always dominated by LDEI, but it is worth here mentioning its existence since it may be relevant in certain astrophysical cases.

\subsection{Typical amplitudes of gravitational distortions}

The amplitude $\beta$ of gravitational distortions, defined here as $\beta=$ $\left|a_{1}^{2}-a_{2}^{2}\right| /\left(a_{1}^{2}+a_{2}^{2}\right)$, where $a_{1}$ and $a_{2}$ are respectively the long and short axes of the outer boundary of the considered fluid layer, is generally unknown for celestial bodies. To study the elliptical instability for real cases, we need to estimate it, for instance by assuming an hydrostatic equilibrium shape.

The equilibrium shape of a body of mass $M$ and radius $R$, is an old problem that begins with the static bulge theory of Newton (1686). This classical theory considers an incompressible no-spinning body at rest, deformed by a tidal field at leading order in $R / D$, which leads to a spheroidal shape and

$\beta=\frac{3}{2} \frac{M_{2}}{M} \frac{R^{3}}{D^{3}}$,

where $M_{2}$ is the mass of the body responsible for the gravitational field and $D$ the distance between the two bodies. This tide is sometimes referred to as the marine tide, where the gravitational potential of the tidal bulge is neglected. This approximation always leads to a relevant but underestimated tidal deformation. When possible, we use in the following a better estimate of $\beta$ that takes the density distribution in the body and the gravitational potential of the tidal bulge into account:

$\beta=\frac{3}{2} h_{2} \frac{M_{2}}{M} \frac{R^{3}}{D^{3}}$

with the radial displacement Love number $h_{2}$, directly linked to the potential Love number $k_{2}$ by $h_{2}=1+k_{2}$. A typical value is $k_{2}=3 / 2$, obtained for an incompressible homogeneous body in hydrostatic equilibrium (e.g. Greff-Lefftz et al. 2005). The tidal Love numbers can be calculated with the Clairaut-Radau theory (see e.g. Van Hoolst et al. 2008).

As shown by Eq. (5), gravitational distortions vary with the interbody distance $D$. They can thus be divided into a component of constant amplitude, corresponding to the mean value of the gravitational distortion along the elliptic orbit, plus a smaller component with an amplitude oscillating between $\pm 3 e$ times the

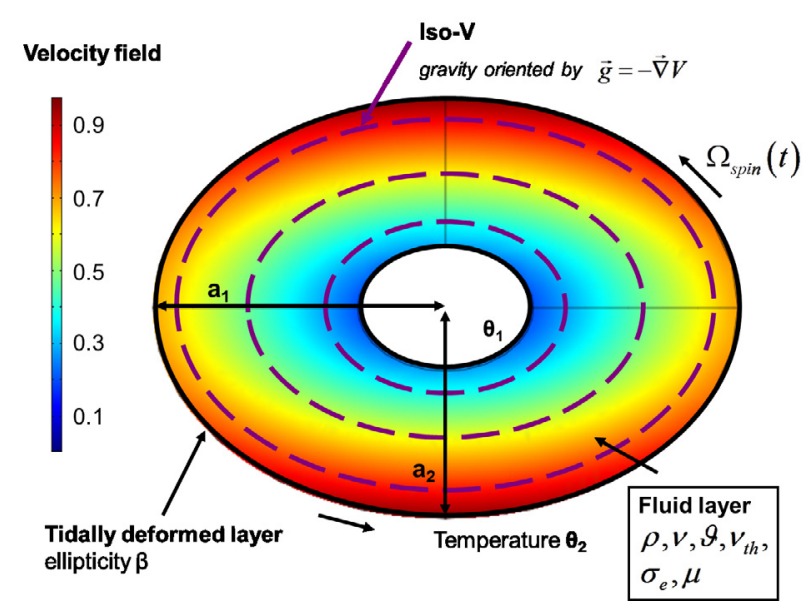

(a)

Fig. 2. Description of an internal liquid layer. The tidal forces deform the core-mantle boundary (CMB) in an ellipse of axes $a_{1}$ and $a_{2}$, which leads to an ellipticity $\beta$ of the streamlines. In this layer, the fluid is rotating at the rate $\Omega$.

constant one (e.g. Greenberg et al. 2003). In real cases that we consider in the following, this oscillating component can be neglected since it will always have a second-order influence on the elliptical instability compared to the constant (static or dynamic) component of $\beta$ (but see Appendix C). Nevertheless, in synchronized satellites, these so-called diurnal tides have important consequences for the internal state and the orbital evolution, since the changing shape of the bulge generates time-varying stresses, which generate heat by viscosity or friction. Besides, as shown by Tobie et al. (2005), these diurnal tides should also induce longitudinal motions in the fluid layers, which constitute a supplementary origin of a non-zero differential rotation between the fluid and the deformation. As already mentioned above, in the context of the present study, this additional component is fully included in the amplitude of the considered physical libration available to drive the elliptical instability.

\section{Generic formulas for the growth rate of the elliptical instability in a planetary context}

\subsection{Model, equations, and dimensionless parameters}

We consider a telluric celestial body in the general framework sketched in Fig. 1, and we focus on a liquid layer described in Fig. 2. All dimensional parameters are listed in Table 2. The instantaneous spin rotation rate $\Omega_{\text {spin }}=\Omega\left(1+\omega_{(t)}\right)$ may depend on time because of either free or forced physical librations. We focus on an internal fluid layer enclosed in an ellipsoidal shell, with an outer boundary of mean radius $R_{2}$ at temperature $\theta_{2}$, and an inner boundary at temperature $\theta_{1}$, with a mean radius $R_{1}=\eta R_{2}$. As already seen above, because of the no spin-up condition, this fluid layer is initially rotating at the constant rate $\Omega$, equal to the mean value of $\Omega_{\text {spin }}$. This layer is considered to be homogeneous, with density $\rho_{0}$, kinematic viscosity $v$, thermal expansion $\vartheta$, thermal diffusivity $v_{\text {th }}$, electrical conductivity $\sigma_{\mathrm{e}}$, and magnetic permeability $\mu$. We focus here on the stability of the elliptical flow in the equatorial plane, but note that our local analysis remains valid in any plane orthogonal to the rotation axis. We choose $R_{2}$ as the length scale and $\Omega^{-1}$ as the time scale so that the mean basic spin of the body has an unit angular velocity along the rotation axis $\left(O, \boldsymbol{e}_{x_{3}}\right)$. The elliptical deformation 
D. Cebron et al.: Elliptical instability in terrestrial planets and moons

Table 2. List of the dimensional variables used in this work.

\begin{tabular}{ll}
\hline \hline Orbital rotation rate & $\Omega_{\text {orb }}(t)$ \\
Spin rotation rate & $\Omega_{\text {spin }}(t)$ \\
Fluid rotation rate* & $\Omega$ \\
Mass of the deformed body & $M$ \\
Mass of the attractor & $M_{2}$ \\
Inter-body distance & $D$ \\
Free libration angular frequency & $\omega_{\text {free }}$ \\
Fluid layer mean external radius & $R_{2}$ \\
Long/short axis in the equatorial plane & $a_{1} / a_{2}$ \\
Fluid layer mean internal radius & $R_{1}=\eta R_{2}$ \\
Imposed external temperature & $\theta_{2}$ \\
Imposed internal temperature & $\theta_{1}$ \\
Gravity at the external radius & $g_{0}$ \\
Imposed magnetic field & $B_{0} \boldsymbol{e}_{\mathrm{x} 3}$ \\
Fluid density & $\rho_{0}$ \\
Fluid kinematic viscosity & $v$ \\
Fluid thermal expansion & $\vartheta$ \\
Fluid thermal diffusivity & $v_{\text {th }}$ \\
Fluid electrical conductivity & $\sigma_{\mathrm{e}}$ \\
Fluid magnetic permeability & $\mu$ \\
\hline
\end{tabular}

Notes. ${ }^{(*)}$ Mean value of the spin rate.

has a dimensionless angular velocity $\gamma_{(t)} \boldsymbol{e}_{\mathrm{x}_{3}}$, with $\gamma_{(t)}$ equal to $\Omega_{\text {orb }}(t) / \Omega$ when looking at dynamic tides and to $\Omega_{\text {spin }}(t) / \Omega$ when looking at static bulges (see Table 1).

We consider the frame where the elliptical distortion is fixed, which is rotating at the angular velocity $\gamma_{(t)} \boldsymbol{e}_{\mathrm{x}_{3}}$, with $\boldsymbol{e}_{\mathrm{x}_{1}}$ in the direction of the long axis $a_{1}$ and $\boldsymbol{e}_{\mathrm{x}_{2}}$ in the direction of the short axis $a_{2}$. The dimensionless equations of fluid motions are

$\nabla \cdot \boldsymbol{u}=0$

$\frac{\partial \boldsymbol{u}}{\partial t}+2 \gamma \boldsymbol{e}_{\mathrm{x}_{3}} \times \boldsymbol{u}+\frac{\mathrm{d} \gamma_{(t)}}{\mathrm{d} t} \boldsymbol{e}_{\mathrm{x}_{3}} \times \boldsymbol{r}+\boldsymbol{u} \cdot \boldsymbol{\nabla} \boldsymbol{u}+\nabla p=E \nabla^{2} \boldsymbol{u}+\boldsymbol{f}$

where $\boldsymbol{u}$ is the fluid velocity; $p$ the pressure (including the centrifugal term) non-dimensionalized by $\rho_{0} R_{2}{ }^{2} \Omega^{2} ; E=v /\left(\Omega R_{2}{ }^{2}\right)$ the Ekman number based on the external radius; and $f=f_{\mathrm{B}}+f_{\mathrm{L}}$ the volumic force, including the buoyancy force $f_{\mathrm{B}}$ and the magnetic Lorentz force $f_{\mathrm{L}}$. The flow is rotating within an ellipse $x_{1}^{2} / a_{1}^{2}+x_{2}^{2} / a_{2}^{2}=1$, and we define the ellipticity as $\beta=$ $\left|a_{1}^{2}-a_{2}^{2}\right| /\left(a_{1}^{2}+a_{2}^{2}\right)$.

Using the dimensionless temperature $\theta=\left(\tilde{\theta}-\theta_{2}\right) /\left(\theta_{1}-\theta_{2}\right)$, the temperature equation is

$\frac{\partial \theta}{\partial t}+(\boldsymbol{u} \cdot \nabla) \theta=\frac{E}{P r}\left(\nabla^{2} \theta-K\right)$

where $\operatorname{Pr}=v / v_{t h}$ is the thermal Prandtl number and $K$ stands for a possible volumic heat source. Considering a gravity $\boldsymbol{g}=g_{(r, \phi)} g_{0} \boldsymbol{e}_{g}$, where $\boldsymbol{e}_{g}$ is a unit vector, $(r, \phi)$ the cylindrical coordinates in the equatorial plane, and $g_{0}$ the gravity at the radius $R_{2}$, the dimensionless buoyancy force to add in the Navier-Stokes equations using the Boussinesq approximation is $\boldsymbol{f}_{\mathrm{B}}=\tilde{R a} \theta g_{(r, \phi)} \boldsymbol{e}_{g}$, with the modified Rayleigh number $\tilde{R} a=$ $\vartheta\left[\theta_{1}-\theta_{2}\right] g_{0} / \Omega^{2} R_{2}$. In a planetary context, the temperature contrast to take into account only corresponds to the non-adiabatic component, which is the deviation from the thermodynamical equilibrium state.

We also take the possible presence of an uniform imposed magnetic field $B_{0}$ along the rotation axis $\boldsymbol{e}_{\mathrm{x}_{3}}$ into account, which
Table 3. List of relevant dimensionless parameters.

\begin{tabular}{ll}
\hline \hline Aspect ratio of the shell & $\eta$ \\
Ellipticity of the distortion & $\beta=\left(a_{1}^{2}-a_{2}^{2}\right) /\left(a_{1}^{2}+a_{2}^{2}\right)$ \\
Distortion rotation rate & $\gamma_{(t)}$ \\
Orbital eccentricity & $e$ \\
Physical libration rate & $\omega_{(t)}$ \\
Physical libration amplitude & $\epsilon$ \\
Volumic heat source & $K$ \\
Ekman number & $E=v /\left(\Omega R_{2}{ }^{2}\right)$ \\
Thermal Prandtl number & $\operatorname{Pr}=v / v_{\text {th }}$ \\
Magnetic Prandtl number & $\operatorname{Pm}=\sigma_{\mathrm{e}} \mu \nu$ \\
Modified Rayleigh number & $\tilde{R a}=\vartheta\left[\theta_{1}-\theta_{2}\right] g_{0} / \Omega^{2} R_{2}$ \\
Magnetic Reynolds number & $\operatorname{Rm}=\sigma_{\mathrm{e}} \mu \Omega R_{2}{ }^{2}$ \\
Elsasser number & $\Lambda=\sigma_{\mathrm{e}} B_{0}^{2} /\left(\rho_{0} \Omega\right)$ \\
\hline
\end{tabular}

is used as the magnetic field scale. The magnetohydrodynamic (MHD) equations then have to be solved simultaneously

$$
\begin{aligned}
\nabla \cdot \boldsymbol{B} & =0 \\
\frac{\partial \boldsymbol{B}}{\partial t}+(\boldsymbol{u} \cdot \nabla) \boldsymbol{B} & =(\boldsymbol{B} \cdot \nabla) \boldsymbol{u}+\frac{1}{R m} \nabla^{2} \boldsymbol{B}
\end{aligned}
$$

with the magnetic Reynolds number $R m=\sigma_{\mathrm{e}} \mu \Omega R_{2}{ }^{2}$. The magnetic Lorentz force acting on the flow is given by $f_{\mathrm{L}}=(\Lambda / R m)(\nabla \times \boldsymbol{B}) \times \boldsymbol{B}$, with the Elsasser number $\Lambda=$ $\sigma_{\mathrm{e}} B_{0}^{2} /\left(\rho_{0} \Omega\right)$. All dimensionless parameters are listed in Table 3.

\subsection{Base fields}

In the reference frame where the elliptical deformation is stationary, the differential rotation of the fluid has an amplitude $1-\gamma_{(t)}$. Besides this, the ellipticity induces an elongational flow $-\left(1-\gamma_{(t)}\right) \beta\left(x_{2} \boldsymbol{e}_{\mathrm{x}_{1}}+x_{1} \boldsymbol{e}_{\mathrm{x}_{2}}\right)$, leading to the general elliptical base flow

$\boldsymbol{U}=\left(1-\gamma_{(t)}\right)\left[-(1+\beta) x_{2} \boldsymbol{e}_{\mathrm{x}_{1}}+(1-\beta) x_{1} \boldsymbol{e}_{\mathrm{x}_{2}}\right]$.

This flow represents the laminar response of the fluid to the tidal distortion as an exact, non-linear solution of Navier-Stokes equations for any finite viscosity, provided that $(\nabla \times f) \cdot \boldsymbol{e}_{3}=2 \mathrm{~d}_{t} \gamma(t)$. This means that a body volumic force noted $f$ is necessary to have spin period fluctuations (i.e. free or forced physical librations), as is obvious in a planetary context. Equation (11) leads to elliptical streamlines of instantaneous ellipticity $\beta$. We note that $\beta$ is not the mathematical eccentricity of the streamlines, given by $\sqrt{2 \beta /(1+\beta)}$. Also the velocity magnitude changes along a streamline, where the isovalues of the velocity are elliptical but with an ellipticity $2 \beta$.

We further assume that a stationary temperature profile $\Theta(r, \phi)$ is imposed, which is, at order 1 in $\beta$, the solution of the energy conservation Eq. (8) with the base field $\boldsymbol{U}$. We suppose that the modified Rayleigh number $\tilde{R a}$ is such that $\tilde{R} a=O(\beta)$. We also consider the presence of an imposed uniform magnetic field along the rotation axis, produced for instance by a companion body. We assume that the Lorentz force does not modify the base flow but only plays a role on the elliptical instability. This implies that this force is $O(\beta)$. In this context, we see below that, regarding the elliptical instability, equations for fluid motions on the order of 0 in $\beta$ are similar to those in the purely hydrodynamical case, where the elliptical instability is described as a resonance between two inertial waves. The magnetic and thermal fields only induce a correction in the fluid equations on the order of 1 in $\beta$, hence a correction of the growth rate of the instability, because of the stabilizing effect of the Lorentz and buoyancy forces. 


\subsection{The WKB method: stability along a streamline}

Our local approach is based on the short-wavelength Lagrangian theory developed in Bayly (1986), Craik \& Criminale (1986), then generalized in Friedlander \& Vishik (1991) and Lifschitz \& Hameiri (1991). This method has been successfully applied to the elliptical instability by Le Dizès (2000), then extended to take the energy equation and the buoyancy force into account (Le Bars \& Le Dizès 2006), or the induction equation and the Lorentz force Herreman et al. (2009). To summarize, the WKB (Wentzel-Kramers-Brillouin) method consists of looking for a perturbed solution of the equations of motion under the form of localized plane waves along the streamlines of the base flow. We thus look for a solution of the linearized non-dimensional system of Eqs. (6)-(10) in the form

$$
\begin{aligned}
\boldsymbol{u}_{(\boldsymbol{x}, t)} & =\boldsymbol{U}+\boldsymbol{u}^{\prime}(t) e^{\mathrm{i} \boldsymbol{k}(t) \cdot \boldsymbol{x}}, \\
\boldsymbol{p}_{(\boldsymbol{x}, t)} & =\boldsymbol{P}+\boldsymbol{p}^{\prime}(t) e^{\mathrm{i} \boldsymbol{k}(t) \cdot \boldsymbol{x}}, \\
\theta_{(\boldsymbol{x}, t)} & =\Theta+\theta^{\prime}(t) e^{\mathrm{i} \boldsymbol{k}(t) \cdot \boldsymbol{x}}, \\
\boldsymbol{B}_{(\boldsymbol{x}, t)} & =\boldsymbol{B}_{0}+\boldsymbol{b}(t) e^{\mathrm{i} \boldsymbol{k}(t) \cdot \boldsymbol{x}},
\end{aligned}
$$

along the streamlines of the base flow described by

$$
\frac{\mathrm{d} \boldsymbol{x}}{\mathrm{d} t}=\boldsymbol{U}
$$

where $\boldsymbol{k}_{(t)}$ is the time-dependent wave vector, $\boldsymbol{x}$ the position vector, and where $\boldsymbol{U}$ (with its corresponding pressure field $\boldsymbol{P}$ ), $\Theta$ and $\boldsymbol{B}_{0}=(0,0,1)$ are the dimensionless base fields defined in Sect. 3.2. Dropping the primes for simplicity, the linearized system of equations writes as

$$
\begin{aligned}
\boldsymbol{k} \cdot \boldsymbol{u}= & 0 \\
\mathrm{~d}_{t} \boldsymbol{u}+ & \mathrm{i} \boldsymbol{u}\left(\mathrm{d}_{t} \boldsymbol{k} \cdot \boldsymbol{x}\right)+\mathrm{i}(\boldsymbol{U} \cdot \boldsymbol{k}) \boldsymbol{u}+(\boldsymbol{u} \cdot \nabla) \boldsymbol{U}+2 \gamma_{(t)} \boldsymbol{e}_{\mathrm{x}_{3}} \times \boldsymbol{u}= \\
& -\mathrm{i} p \boldsymbol{k}-k^{2} E \boldsymbol{u}+\frac{\Lambda}{R m}(\mathrm{i} \boldsymbol{k} \times \boldsymbol{b}) \times \boldsymbol{B}_{0}+\tilde{R a} \theta g \boldsymbol{e}_{g} \\
\mathrm{~d}_{t} \theta+ & \mathrm{i} \theta\left(\mathrm{d}_{t} \boldsymbol{k} \cdot \boldsymbol{x}\right)+\mathrm{i}(\boldsymbol{U} \cdot \boldsymbol{k}) \theta+(\boldsymbol{u} \cdot \nabla) \boldsymbol{\Theta}=-k^{2} \frac{E}{P r} \theta \\
\boldsymbol{k} \cdot \boldsymbol{b}= & 0 \\
\mathrm{~d}_{t} \boldsymbol{b}+ & \mathrm{i} \boldsymbol{b}\left(\mathrm{d}_{t} \boldsymbol{k} \cdot \boldsymbol{x}\right)+\mathrm{i}(\boldsymbol{U} \cdot \boldsymbol{k}) \boldsymbol{b}= \\
& (\boldsymbol{b} \cdot \nabla) \boldsymbol{U}+\mathrm{i}\left(\boldsymbol{B}_{0} \cdot \boldsymbol{k}\right) \boldsymbol{u}-\frac{k^{2}}{R m} \boldsymbol{b} .
\end{aligned}
$$

Those equations can be decoupled in space and time to give an equation for the wave vector only:

$\mathrm{d}_{t} \boldsymbol{k} \cdot \boldsymbol{x}+\boldsymbol{U} \cdot \boldsymbol{k}=0$.

The solution of the remaining equations for $\boldsymbol{u}, \theta, \boldsymbol{b}$ is then sought under the form of a Taylor expansion in $\beta$ of all variables, as illustrated in Appendices A and B.

This approach is used in the following sections to calculate the growth rate of the instability in the two generic cases: the TDEI, which appears in the case of non-synchronized bodies, and the LDEI, which appears in the case of synchronized bodies.

\subsection{Non-synchronized bodies: inviscid growth rate of the TDEI}

In this section, we consider the effects of dynamic tides of amplitude $\beta$ on the liquid core of a Mercury-like planet orbiting close to its star with (i) constant but different orbital and sidereal rotation periods, (ii) an imposed thermal stratification (see e.g. Manglik et al. 2010), and (iii) an externally imposed magnetic field (e.g. the Sun magnetic field). The same analysis applies to the stratified zone of a star (the so-called radiative zone) tidally deformed by a companion body, taking the magnetic field generated by dynamo in its convective zone into account. The present configuration corresponds to the standard case of the elliptical instability as already known, but completed by the complexities present in real astrophysical cases. These additive effects have already been studied separately, even if they are simultaneously present in real systems. The effect of the angular velocity of the tidal bulge has been studied in Miyazaki \& Fukumoto (1992), Le Dizès (2000), Le Bars et al. (2007) and Le Bars et al. (2010) and the presence of a thermal field has been studied in Le Bars \& Le Dizès (2006), who study the linear competition between the growths of the TDEI and the convection, as well as in Cébron et al. (2010c) and Lavorel \& Le Bars (2010), who study the growth of the TDEI over established convective flows. The presence of an inner solid core has been studied in Lacaze et al. (2005) and of an external magnetic field in Kerswell (1994), Kerswell (2002), Lacaze et al. (2006) and Herreman et al. (2009). We extend these works by including all of these features in a single formula.

In the non-synchronized case, which is considered in this section, the base flow (11) reduces to

$\boldsymbol{U}=\left(1-\Omega_{\text {orb }} / \Omega_{\text {spin }}\right)\left[-(1+\beta) x_{2} \boldsymbol{e}_{\mathrm{x}_{1}}+(1-\beta) x_{1} \boldsymbol{e}_{\mathrm{x}_{2}}\right]$.

The WKB analysis is then tractable (see Appendix A), taking thermal and magnetic effects into account in the limit where buoyancy and Lorentz forces are on the order of $\beta$. The instability does not exist in the range $\Omega_{\text {spin }} / \Omega_{\text {orb }} \in[-1 ; 1 / 3]$, which is called the forbidden band. It corresponds to the absence of resonance between the elliptical forcing and the inertial waves of the rotating flow (see Le Bars et al. 2007, for a complete discussion). In the present limit, the presence of the thermal and magnetic fields does not affect the forbidden band. Neglecting the thermal diffusion, the inviscid growth rate of the TDEI with the presence of thermal and magnetic fields is

$\sigma_{\text {inv }}=\frac{\sqrt{\left(2 \Omega^{G}+3\right)^{4} \beta^{2}-4\left(\tilde{\operatorname{Ra}} r \partial_{r} \Theta\right)^{2}}}{16\left|1+\Omega^{G}\right|^{3}}-\frac{\Lambda}{4\left|1+\Omega^{G}\right|^{3}}$,

with $\Omega^{G}=\Omega_{\text {orb }} /\left(\Omega_{\text {spin }}-\Omega_{\text {orb }}\right), r$ the radius and $\partial_{r} \theta$ the dimensionless temperature base-field radial gradient on the considered streamline. This expression allows to recover the different cases already obtained in the literature. For instance, the purely hydrodynamic growth rate given by Miyazaki \& Fukumoto (1992), Le Dizès (2000), and Le Bars et al. (2010) is recovered for $(\tilde{R a}=0, \Lambda=0)$. For a fixed elliptical deformation, we recover the classical inviscid value $\sigma_{\text {inv }} / \beta=9 / 16$. Finally, in the absence of a thermal field and with a stationary bulge $\left(\Omega_{\text {orb }}=0, \tilde{R a}=0\right)$, the magnetic case given in Herreman et al. (2009) is also recovered. Formula (24) is fully generic and clearly illustrates the stabilizing influence of Joule dissipation and of a local stratification in the range of validity of this stability analysis (see Sect. 3.7 and Appendix D).

\subsection{Synchronized bodies: inviscid growth rate of the LDEI}

In this section, we consider the liquid ellipsoidal core of a synchronized moon like Io or of an extrasolar telluric planet orbiting close to its massive attractor with (i) an orbital period equal to the sidereal rotation period, but with small instantaneous fluctuations of the differential rotation between the elliptical deformation and the fluid, whatever their origin (optical 
or physical, forced or free libration, longitudinal flows induced by tides); (ii) an imposed magnetic field (e.g. Jupiter's magnetic field for Io); and (iii) a local thermal gradient. The dimensionless instantaneous differential rotation between the fluid and the elliptic deformation oscillates with a libration amplitude $\epsilon$ (equal to $2 e$ for optical librations) and a libration frequency $\omega_{\mathrm{o}}$ (equal to 1 for forced librations). Considering the particular case of a fluctuation due to the orbital ellipticity (i.e. $\omega_{0}=1$ ), Kerswell $\&$ Malkus (1998) and Herreman et al. (2009) have shown that these oscillations can lead to LDEI. We extend these previous studies to the more general case of small fluctuations of arbitrary periods, taking buoyancy into account. Neglecting the thermal diffusion, the inviscid growth rate of the LDEI is then (see Appendix B)

$\sigma_{\text {inv }}=\frac{16+\omega_{\mathrm{o}}^{2}}{64} \sqrt{(\epsilon \beta)^{2}-\frac{4}{\omega_{\mathrm{o}}^{2}}\left(\tilde{\operatorname{Ra}} r \partial_{r} \Theta\right)^{2}}-\frac{\omega_{\mathrm{o}}^{2}}{16} \Lambda$

at first order in $\epsilon \beta$, taking the effects of thermal and magnetic fields into account in the limit where buoyancy and Lorentz forces are $O(\beta)$. The forbidden band is given by $\left|\omega_{0}\right|>4$. As before, the generic formula (25) clearly illustrates the stabilizing influence of Joule dissipation and of a local stratification.

The case $\omega_{0} \rightarrow 0$ corresponds to the limit toward the fully synchronized state. In the case of the TDEI, this limit case is obtained with $\Omega^{G} \rightarrow \infty$, which gives the inviscid growth rate $\sigma_{\text {inv }}=\beta /\left(4 \Omega^{G}\right)$ for large wavenumbers. Both expressions for the growth rate are thus consistent in the limit of synchronized state: $\Omega_{\text {orb }} / \Omega_{\text {spin }}=1-\epsilon$ i.e. $\left|1+\Omega^{G}\right| \sim\left|\Omega^{G}\right|=1 / \epsilon$. The expression given in Herreman et al. (2009) is also exactly recovered when $\omega_{0}=1$ and $\Lambda=0$. There is a slight error on the numerator of the magnetic damping term in Herreman et al. (2009): in their considered case, $\omega_{0}=1$, the magnetic damping is erroneously $-\Lambda / 16$, instead of $-3 \Lambda / 16$.

\subsection{Viscous dissipation}

The previous sections present the calculation of the growth rate of the elliptic instability in an inviscid fluid with Joule dissipation and buoyancy stabilization. Calculation of the threshold of the instability requires correctly estimating all dissipative terms. In the case of no-slip boundaries, dissipation occurs mainly in the viscous boundary layers of thickness $E^{1 / 2}$. This implies a damping term that should alter the growth rate:

$\sigma=\sigma_{\text {inv }}-\alpha E^{1 / 2} f(\eta)$

where $\alpha$ is a constant between 1 and 10, equal to $\alpha=2.62$ and $f(\eta)=\left(1+\eta^{4}\right) /\left(1-\eta^{5}\right)$ for the spinover mode of the TDEI (see e.g. Kudlick 1966; Hollerbach \& Kerswell 1995; Lacaze et al. 2005).

In addition to decreasing the growth rate, viscous dissipation is also primordial for quantifying the orbital evolution and rotational history of a binary system during its synchronization. A model has been proposed in Le Bars et al. (2010) for $\Omega_{\mathrm{orb}}=0$, which allows the authors to estimate the viscous power dissipated by TDEI. Our purpose here is to generalize this model to all cases studied above. Far from threshold, the model proposed by Le Bars et al. (2010) considers that the TDEI simply corresponds to a differential rotation between the boundary and the bulk. According to this model, the power dissipated by the system is

$$
P=-2 M R_{2}^{2} \triangle \Omega^{2} \Omega E^{1 / 2},
$$

assuming that in the small Ekman numbers limit reached in astrophysical cases, the amplitude of the instability is commensurate with the differential rotation $\Delta \Omega$ (Cébron et al. 2010a).

The tidal quality factor $Q$ is widely used in systems evolution calculations. By analogy with the theory of harmonic oscillators, $Q$ is defined by (e.g. Greenberg 2009, for a recent discussion on $Q$ ) the ratio between the maximum potential gravitational energy stored in the tidal distortion over the energy dissipated in one work cycle. The dissipated power associated to the flow driven by the elliptical instability does not have the periodicity of the forcing, so a quality factor cannot be rigorously defined in the same way. However, we can define a closely related ratio $Q^{*}$, comparing the power typically dissipated in the fluid layer over one revolution with the potential energy stored in the bulge, which is on the order of $E_{0} \sim 4 \pi \rho_{0} g_{0} s^{2} R_{2}^{2}$, with $s$ the dimensional height of the tides (e.g. Benest 1990). Since $\beta \sim s / R_{2}$, we obtain from Eq. (27)

$Q^{*} \sim \frac{g_{0} \beta^{2}}{R \triangle \Omega^{2} E^{1 / 2}}$.

The ratio $Q^{*}$ gives a dimensionless measure of the strength of the dissipation in the fluid.

\subsection{Validity of the approach}

The previous analysis is valid when the elliptic instability comes from a resonance of pure hydrodynamic inertial waves. Therefore, any previously derived expressions are limited to the case where buoyancy and Lorentz forces are $O(\beta)$. According to (17), this means that

$\frac{\Lambda}{R m} k \sim \beta \quad$ and $\quad \tilde{R} a \sim \beta$,

where $k$ is the dimensionless norm of the wavevector of the excited mode. For a typical planetary core, these conditions can be rewritten

$$
B_{0} \sim 0.1 \frac{\sqrt{\beta / k}}{R_{2} E} \quad \text { in } \mu \mathrm{T} \text { and } \frac{F_{\text {non-adia }}}{F_{\text {adia }}} \sim 10^{-3} \frac{\beta}{E^{2} R_{2}^{4} g_{0}^{2}},
$$

where $F_{\text {non-adia }}$ and $F_{\text {adia }}$ are the non-adiabatic and adiabatic components of the core heat flux, respectively. The condition on the magnetic field is easily verified for planetary cores over a wide range of wave vector $k$. The condition on the non-adiabatic heat flux is more problematic to quantify: in most planets, the adiabatic profile is supposed to be sufficient to transport core heat flux, and the non-adiabatic component is estimated to be very small, but it is not known precisely. One should notice that the condition (30) is very restrictive, and special attention should be paid in each given configuration. For instance, in the case of Europa, Eq. (30) implies $F_{\text {non-adia }} / F_{\text {adia }} \sim 0.1 \%$, which seems reasonable; but in the case of Io, it implies $F_{\text {non-adia }} / F_{\text {adia }} \sim$ $1 \%$, which is only marginally verified since the estimated nonadiabatic heat flux is about one fifth of the adiabatic component (e.g. Kerswell \& Malkus 1998).

Now supposing that the buoyancy or the Lorentz force is on the order of 0 in $\beta$, we can wonder if the elliptic instability still exists. In this case, inertial waves are replaced by gravitoinertial or magneto-inertial waves, and the elliptical instability arises as a resonance between those modified waves. Resonances of magneto-inertial waves has been studied, for instance, in Kerswell (1994), Lebovitz \& Zweibel (2004) and Mizerski \& Bajer (2009) in the case of an imposed uniform magnetic field 
Table 4. Physical and orbital characteristics used for the stability calculations and results.

\begin{tabular}{lcccc}
\hline \hline & Mercury & Venus & Earth & Early Earth $^{b}$ \\
\hline$M\left(\times 10^{-24} \mathrm{~kg}\right)$ & 0.330 & 4.87 & 5.98 & 5.98 \\
$R(\mathrm{~km})$ & 2440 & 6051 & 6378 & 6378 \\
$T_{\text {spin }}(d)$ & 58.6 & -243 & 0.997 & 0.418 \\
$T_{\text {orb }}(d)$ & 87.97 & 224.7 & 27.32 & $9.67^{b}$ \\
Tidal amplitude $(\mathrm{m})$ & $0.925^{a}$ & 1.8 & 0.6 & 3.4 \\
$R_{2} / R$ & $0.8^{a}$ & 0.17 & 0.55 & 0.55 \\
$\eta$ & 0 & 0 & 0.35 & 0 \\
$E\left(\times 10^{14}\right)$ & 21 & 316 & 0.11 & 0.047 \\
$\beta($ measured $)\left(\times 10^{7}\right)$ & 7.6 & $?$ & 1.9 & 11 \\
$\beta$ (hydrostatic) $\left(\times 10^{7}\right)$ & 6.8 & 1.1 & 0.8 & 6.7 \\
$B_{\text {suf }}{ }^{c}(\mathrm{nT})$ & $250^{d}$ & 30 & $3 \times 10^{4}$ & 0 \\
$B_{0}{ }^{e}(\mathrm{nT})$ & 488 & 6100 & $1.8 \times 10^{5}$ & 0 \\
$\Lambda$ & $6.4 \times 10^{-6}$ & 0.004 & 0.015 & 0 \\
$\sigma\left(\right.$ years $\left.^{-1}\right)$ & $-1.5 \times 10^{-5}$ & -5.43 & -7.7 & 0.003 \\
\hline
\end{tabular}

Notes. Following Herreman et al. (2009), we take as typical values $\sigma_{\mathrm{e}}=$ $4 \times 10^{5} \mathrm{~S} \mathrm{~m}^{-1}, \rho_{0}=12000 \mathrm{~kg} \mathrm{~m}^{-3}$ and $v=10^{-6} \mathrm{~m}^{2} \mathrm{~s}^{-1}$, consistent with a Fe/Fe-S composition. ${ }^{(a)}$ Van Hoolst et al. (2007). ${ }^{(b)}$ Considering an Early Moon two times closer than today. ${ }^{(c)}$ Equatorial surface field. (d) Anderson et al. (2010). ${ }^{(e)}$ Considering a variation in $r^{-3}$ from the core to the planetary surface ( $r$ being the spherical radius).

along the spin axis. This is the so-called magneto-elliptic instability. Resonance of gravito-inertial waves has been studied in Le Bars \& Le Dizès (2006) and Guimbard et al. (2010), who concluded that a stratified field can either be stabilizing or destabilizing depending on the shape of gravitational isopotentials and isotherms: the so-called gravito-elliptic instability. This point is further clarified in appendix D, which shows the high sensitivity of the elliptical instability to the specificities of the thermal and gravity fields. Planets with buoyancy or Lorentz force on the order of 0 in $\beta$ should be the subject of specific studies, which is beyond the scope of the generic results presented here.

\section{Application to solar/extrasolar systems}

Using previous results, we are now in a position to calculate the threshold of the elliptical instability for telluric bodies of different systems. We consider that a body is stable or unstable when the mean value of the growth rate over an orbit is either positive or negative.

\subsection{Non-synchronized system: tide-driven elliptical instability (TDEI)}

We consider first the TDEI in liquid cores of telluric bodies in the solar system. A rough criteria given by Eqs. (24) and (26) leads to a threshold $\beta / \sqrt{E} \sim O(1)$, as already mentioned. In the solar system, this leads to focus only on Mercury, Venus, and on the Earth-Moon system during its evolution. Most tidal evolution models predict that the Moon rapidly retreats to 25-35 Earth radii in less than about $100 \mathrm{Ma}$ (Webb 1982; Ross \& Schubert 1989; Williams 2000, 2004), and we thus consider two limit cases: the actual Earth-Moon system and an early Earth with an early Moon at 30 Earth radii, i.e. two times closer than today. The tabulated values found in the literature for these planets are given in Table 4. The Ekman number $E$ is calculated with a molecular kinematic viscosity $v=10^{-6} \mathrm{~m}^{2} \mathrm{~s}^{-1}$, consistent with $\mathrm{Fe} / \mathrm{Fe}-\mathrm{S}$ composition of a liquid outer core.

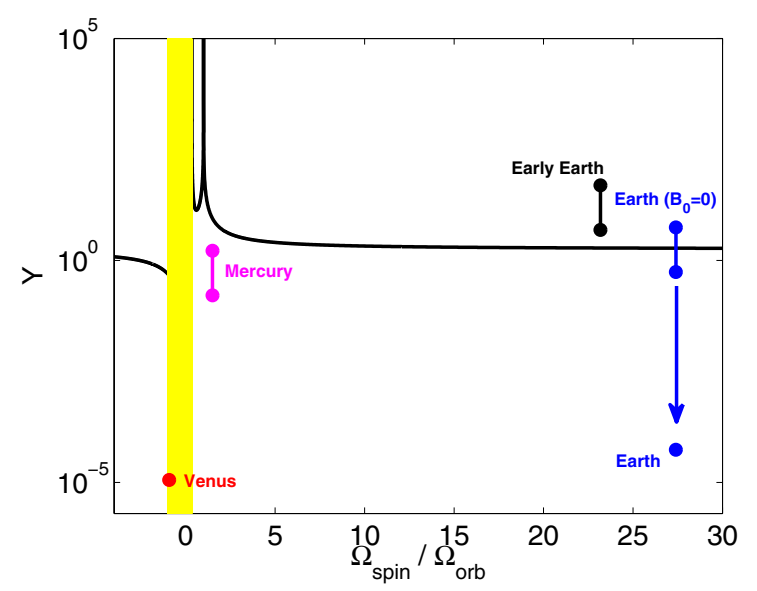

Fig. 3. TDEI stability diagram for celestial bodies of the solar system. Considering a surfacic viscous damping term of the growth rate $\sigma_{\mathrm{s}}=$ $-\alpha f(\eta) E^{1 / 2}$ (see Sect. 3.6), the zone above the black line, defined by (33), is the unstable zone, whereas $Y$, defined by (31), is calculated with $\alpha=1$ and $\alpha=10$ for each planet (for the actual Earth and Venus, the difference is very small). The yellow zone is the so-called "forbidden zone", given by $\Omega_{\text {spin }} / \Omega_{\text {orb }} \in[-1 ; 1 / 3]$.

We first neglect thermal effects. To represent all bodies of Table 4 on the same stability diagram, we define the quantity

$Y=\beta\left(\alpha f(\eta) \sqrt{E}+\frac{\Lambda}{4\left|1+\Omega^{G}\right|^{3}}\right)^{-1}$.

The quantity $Y$ includes the specific dependence of the growth rate on the spin/orbit angular velocity ratio, aspect ratio $\eta$ of the inner core and the magnetic field. The stability criterium

$\sigma=\frac{\left(2 \Omega^{G}+3\right)^{2}}{16\left|1+\Omega^{G}\right|^{3}} \beta-\alpha f_{(\eta)} \sqrt{E}-\frac{\Lambda}{4\left|1+\Omega^{G}\right|^{3}} \geq 0$

derived from (24) and (26) is then equivalent to

$Y \geq \frac{16\left|1+\Omega^{G}\right|^{3}}{\left(3+2 \Omega^{G}\right)^{2}}$.

Figure 3 represents the stability results for the TDEI in the liquid cores of the non-synchronized planets of the solar system considered in Table 4 . The case of each planet is discussed in the following.

An important result, already noticed in Cébron et al. (2010a), is that the early Earth, with a Moon two times closer than today and in the absence of an external magnetic field, is unstable with a good level of confidence. The dissipated power due to the instability was around $5 \times 10^{18} \mathrm{~W}$, which corresponds to $Q^{*} \sim 0.003$. This estimation seems huge in comparison to the present dissipation by tidal friction $\left(\sim 3.75 \times 10^{12} \mathrm{~W}\right.$ according to Munk \& Wunsch 1998). However, one must notice that the estimations given here are based on a model where the rotation rate is implicitly expected to be constant, corresponding to a quasi-static approximation of the orbital evolution of the system. This approximation obviously breaks down for a high dissipation rate, and the above result should be interpreted as proof of a rapid orbital evolution of the Earth-Moon system. The Moon orbit inclination is not taken into account in the stability analysis considered in this work. A similar analysis would be difficult because the forced base flow in such a configuration is not analytically known. However, it has been demonstrated numerically in Cébron et al. (2010a) that this inclination only has minor consequences on the process of elliptical instability, which may be 


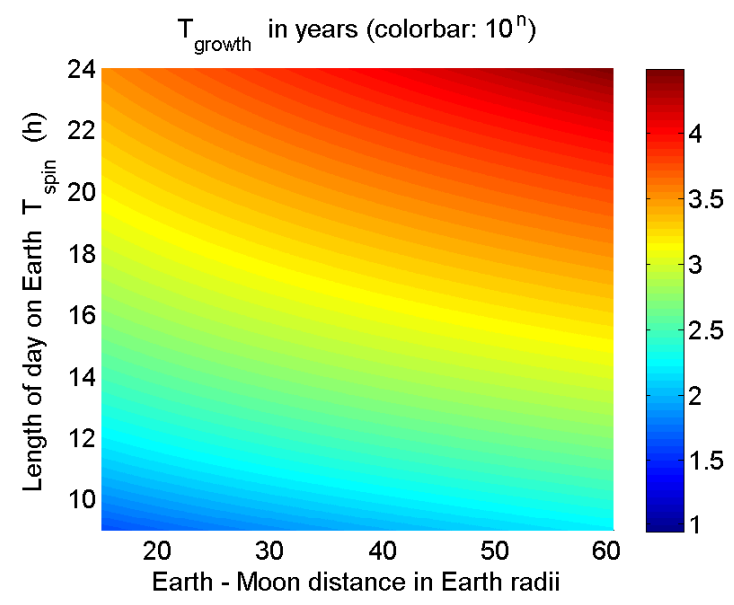

Fig. 4. Evolution of the typical growth time $T_{\text {growth }}=1 / \sigma$ of the instability in the early Earth core as a function of the Earth-Moon separation and of the length of day on the early Earth $T_{\text {spin }}(\alpha=2.62)$. This diagram assumes no external magnetic field $\left(B_{0}=0\right)$ and no thermal field $(\tilde{R} a=0)$.

easily taken into account in considering an effective elliptical distortion in the equatorial plane. In the present case, this orbital inclination would slightly decrease our growth rates but does not change the orders of magnitude our conclusions either.

Figure 4 shows the stability of the early Earth in more detail, for different values for the length of day and Earth-Moon distances. In the absence of meteoroid impacts, the angular momentum conservation links these two quantities, which cannot vary independently. However, at this epoch, violent meteoroid impacts have probably modified the angular momentum of the early Earth-Moon system (Melosh 1975; Wieczorek \& Le Feuvre 2009), so we keep these two parameters independent, which allows us to cope with uncertainties.

The case of the actual Earth is more subtle because if we consider that the actual magnetic field is provided by thermosolutal convective motions in the core, it has to be considered as an imposed field for the dynamics of the elliptical instability. In this case, the destabilizing term in the growth rate (32) is about $10^{-7}$, whereas the magnetic damping term is around 0.004 . Then the TDEI cannot grow, regardeless of the Ekman number. On the contrary, if we consider that the actual magnetic field is provided by the flow driven by the TDEI, the threshold has to be calculated with $\Lambda=0$, and the actual Earth is slightly unstable, with a growth time of around 14,000 years. The same result was suggested by Aldridge et al. (1997), but neglecting the influence of magnetic field and global rotation.

We now consider the influence of thermal effects. Considering the actual heat flux of Earth, Christensen \& Aubert (2006) estimate the corresponding super-adiabatic temperature contrast to be about $1 \mathrm{mK}$, leading to a vigorous convection in the liquid core. Such a vigorous convection does not prevent the elliptical instability from growing, as shown in Cébron et al. (2010c); Lavorel \& Le Bars (2010). Since this temperature contrast is uncertain, we can consider as an extreme case for stabilization of the TDEI, a subadiabatic gradient on the same order of magnitude. For the actual Earth, such a stratification leads to $\tilde{R a} \approx-1.4 \times 10^{-6}$, and the dependence $\tilde{R a} \propto D^{3} E^{2}$ gives the value $\tilde{R a} \approx-2 \times 10^{-7}$ for the early Earth (see Sumita \& Yoshida 2003, for a discussion of this possible stable density stratification in the whole early Earth outer core and its disruption). Considering $\tilde{R} a \approx-1 \times 10^{-6}$ as an upper bound, formula (24)
Table 5. Physical and orbital characteristics used for the four Galilean moons and Titan.

\begin{tabular}{lccccc}
\hline \hline & Io & Europa & Ganymede & Callisto & Titan \\
\hline$M\left(\times 10^{-22} \mathrm{~kg}\right)$ & 8.93 & 4.8 & 14.8 & 10.8 & 13.45 \\
$R(\mathrm{~km})$ & 1822 & 1561 & 2631 & 2410 & 2576 \\
$T_{\text {orb }}(d)$ & 1.77 & 3.55 & 7.16 & 16.69 & 15.95 \\
$e\left(\times 10^{3}\right)$ & 4.1 & 9.4 & 1.3 & 7.4 & 28.8 \\
$\epsilon\left(\times 10^{4}\right)$ & $1.3^{a}$ & $2^{a}$ & $0.056^{a}$ & $0.042^{a}$ & $1.3^{a}$ \\
$B_{0}(\mathrm{nT})$ & 1850 & $410^{b}$ & 120 & $10^{b}$ & 0 \\
\hline
\end{tabular}

Notes. ${ }^{(a)}$ Physical libration amplitude from Noir et al. (2009). ${ }^{(b)}$ Order of magnitude from Zimmer et al. (2000) of the magnetic field component along the rotation axis of the moon (see also Kabin et al. 1999).

is valid $(\tilde{R} a \sim O(\beta))$ and shows that the thermal stratification reduces the growth rate by $2 \%$. This confirms that the role of the temperature can be neglected in the limits considered in this work.

For the last two bodies, Venus is in the forbidden band, which means that no matter what the tidal deformation or the Ekman number is, the TDEI cannot grow. Mercury is slightly below the threshold of the instability and is thus probably stable today. Mars is clearly stable nowadays $(\beta \ll \sqrt{E})$, but Arkani-Hamed et al. (2008) and Arkani-Hamed (2009) suggest that past gravitational interactions with asteroids could have excited a TDEI in the martian core during their fall towards the planet.

\subsection{Synchronized body: libration-driven elliptical instability (LDEI)}

\subsubsection{Galilean moons and Titan}

The presence of LDEI in Io has been first suggested by Kerswell \& Malkus (1998). In Herreman et al. (2009), this suggestion is reexamined and the magnetic field induced by this possible instability quantified. In the following, this calculation is reevaluated and extended to the four Galilean moons (Io, Europa, Ganymede, and Callisto), considering the presence of the external magnetic field of Jupiter. Titan is also considered. All necessary data are given in Tables 5 and 6 .

As described in Sect. 3.5, we consider an instantaneous differential rotation $\epsilon \cos \left(\omega_{0} t\right)$ for these synchronized bodies. Focusing on the forced librations due to the orbital eccentricity, the libration frequency is $\omega_{0}=1$. The amplitude of the libration $\epsilon$ is given by $\epsilon=2 e$ for optical librations. For physical librations, obtained for $\Re \gg 1, \epsilon$ has to be measured or estimated, but are less than the extreme value $2 e$ (see the data in Noir et al. 2009). The theoretical analysis is the same, and the use of formula (25) with $\tilde{R a}=0$ (thermal field negligible) and $\omega_{\mathrm{o}}=1$ gives the LDEI threshold. To obtain a unique stability diagram for all bodies in Table 5, we define the quantity

$Y_{2}=\left[\epsilon \beta-\frac{4}{17} \Lambda\right]\left[\alpha(1-\eta) f_{(\eta)}\right]^{-1}$

and use the Ekman number based on the thickness $E_{k}=E /(1-$ $\eta)^{2}$. The threshold for LDEI given by formulas (25) and (26)

$\sigma=\frac{17}{64} \epsilon \beta-\alpha(1-\eta) f_{(\eta)} \sqrt{E_{k}}-\frac{1}{16} \Lambda \geq 0$

is then equivalent to

$Y_{2} \geq \frac{64}{17} \sqrt{E_{k}}$ 
Table 6. Stability results in the extreme case of optical librations $(\epsilon=2 e$ and $\beta$ from this table and Table 5).

\begin{tabular}{lccccc}
\hline \hline & Io & Europa & Ganymede & Callisto & Titan \\
\hline & core & core & core & core & core \\
$R_{2} / R$ & $0.52^{a, b}$ & $0.38^{b}$ & $0.27^{c}$ & - & - \\
$\eta$ & 0 & 0 & 0 & - & - \\
$E\left(\times 10^{14}\right)$ & 2.7 & 14 & 20 & - & - \\
$\beta\left(\times 10^{4}\right)$ & $60^{a}$ & $9.7^{d}$ & $3.7^{e}$ & - & - \\
$\Lambda\left(\times 10^{7}\right)$ & 42 & 4.1 & 0.7 & - & - \\
$\sigma\left(\mathrm{yr}^{-1}\right)$ & 0.016 & 0.0025 & $-3 \times 10^{-4}$ & - & - \\
& & & & & \\
& ocean & ocean & ocean & ocean & ocean \\
Crust $(\mathrm{km})$ & - & $10^{g}$ & $100^{h}$ & $150^{i}$ & $70^{j}$ \\
Depth $(\mathrm{km})$ & - & $100^{g}$ & $150^{h}$ & $150^{i}$ & $200^{j}$ \\
$R_{2} / R$ & - & 0.99 & 0.96 & 0.94 & 0.97 \\
$\eta$ & - & 0.94 & 0.94 & 0.93 & 0.92 \\
$E\left(\times 10^{14}\right)$ & - & 2.0 & 1.5 & 4.5 & 3.5 \\
$\beta\left(\times 10^{4}\right)$ & - & $9.7^{d}$ & $3.7^{e}$ & $0.72^{e}$ & $1.2^{f}$ \\
$\Lambda\left(\times 10^{13}\right)$ & - & 21 & 3.5 & 0.9 & 0 \\
$\sigma\left(\mathrm{yr}^{-1}\right)$ & - & 0.0016 & $-6 \times 10^{-4}$ & $-4 \times 10^{-4}$ & $-10^{-4}$ \\
\hline
\end{tabular}

Notes. For the liquid cores, we take $\sigma_{\mathrm{e}}=4 \times 10^{5} \mathrm{~S}_{\mathrm{S}} \mathrm{m}^{-1}, \rho_{0}=$ $8000 \mathrm{~kg} \mathrm{~m}^{-3}$ and $v=10^{-6} \mathrm{~m}^{2} \mathrm{~s}^{-1}$ as typical values, consistent with a $\mathrm{Fe} / \mathrm{Fe}-\mathrm{S}$ composition. For the subsurface oceans, we take $\sigma_{\mathrm{e}}=$ $0.25 \mathrm{~S} \mathrm{~m}^{-1}$ (Hand \& Chyba 2007), $\rho_{0}=1000 \mathrm{~kg} \mathrm{~m}^{-3}$, and $v=$ $10^{-6} \mathrm{~m}^{2} \mathrm{~s}^{-1}$ as typical values. ${ }^{(a)}$ Kerswell \& Malkus (1998), considering the static tidal bulge. ${ }^{(b)}$ Hussmann \& Spohn (2004). ${ }^{(c)}$ Bland et al. (2008). ${ }^{(d)}$ with $k_{2} \approx 0.3$ (Wahr et al. 2006; Baland \& Van Hoolst 2010). ${ }^{(e)}$ Equation (5) with $k_{2} \approx 0.3$. ${ }^{(f)} k_{2} \approx 1$ (Goldreich \& Mitchell 2010). (g) Wahr et al. (2006). ${ }^{(h)}$ Bland et al. (2009). ${ }^{(i)}$ Kuskov \& Kronrod (2005). ${ }^{(j)}$ Sohl et al. (2003).

This allows us to plot the stability diagram shown in Fig. 5 in the extreme case of optical libration $(\epsilon=2 e)$ for a quasiequilibrium hydrostatic bulge calculated with formula (5), corresponding to the optimal case for LDEI (i.e. the maximum possible libration amplitude and the maximum possible elliptical deformation). In the following, we discuss the stability versus the LDEI of the Galilean moons, Titan, and three Super-Earths. Because the tidal bulge and the libration amplitudes of these bodies are not yet known (see the discussion of Goldreich \& Mitchell 2010), we present the results in Figs. 6-8 on diagrams in the $(\beta, \epsilon)$ plane, taking the full range of variability of $\beta$ and $\epsilon$ into account. Therefore, the upper right hand corner will correspond to the optimal case for the LDEI: the libration of a purely deformable body i.e. optical libration with $\epsilon=2 e$ and an hydrostatic bulge. The lower left hand corner corresponds to the libration of a rigid body (physical librations), associated with the weak diurnal tides. In the same way, the lower right hand corner corresponds also to physical librations, but with a hydrostatic bulge. Finally, the upper left hand corner corresponds to the libration of a purely deformable body (optical libration) associated to the small diurnal tides amplitude. The relevant physical configurations for each body depend on their compositions so is specifically discussed in the following for each of them.

First, we consider Io with the values used in the studies of Kerswell \& Malkus (1998) and Herreman et al. (2009), i.e. a static bulge of ellipticity $\beta=0.006$ and a libration amplitude assumed to be $\epsilon=2 e$ (see Table 5). As already found by these authors, Io is unstable with a good level of confidence (typical growth time of 63 years). However, this is an optimal unrealistic case, because the ellipticity used is due to a static bulge $(\Re \gg 1)$, and the libration amplitude is taken as equal to $2 e$, as in the deformable case $(\Re \ll 1)$. Due to its silicate mantle, the

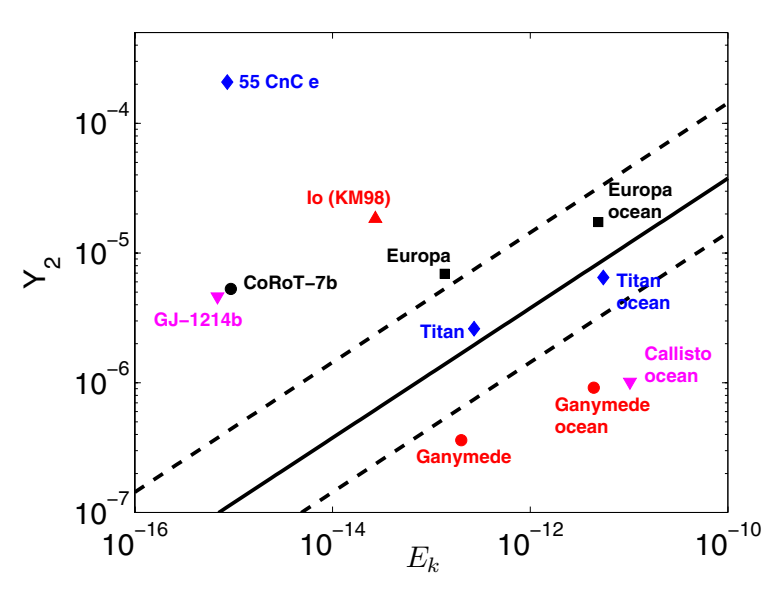

Fig. 5. LDEI stability diagram for synchronized celestial bodies in the optimal case for the instability, i.e. an optical libration $(\epsilon=2 e)$ for a quasi-equilibrium tide. All values are given in Table 5, and the horizontal axis represents the Ekman number $E_{k}$ based on the thickness of the fluid layer. The label KM98 for Io calls that the point is placed with the values used by Kerswell \& Malkus (1998). The zone below the black line, defined by the viscous surfacic damping coefficient $\alpha=2.62$, is the stable zone, whereas the black dashed lines represent the extremum values $\alpha=1$ and $\alpha=10$.

core of Io is expected to be in the limit $\Re \gg 1$, and consequently the ellipticity to consider is indeed $\beta=0.006$ but the libration amplitude is instead $\epsilon \approx 1.3 \times 10^{-4}$ (see Table 5), which is 63 times smaller than $2 e$. With these more realistic values and condidering the presence of Jupiter magnetic field, Io is expected to be stable, unlike what was expected. However, to obtain a better view of the stability in Io, Fig. 6 gives the typical growth time of the instability for different ellipticities and libration amplitudes, ranging between the limit cases $\mathfrak{R} \gg 1$ and $\mathfrak{R} \ll 1$, with $\epsilon=2 e$ and the diurnal tidal ellipticity $3 e \beta \approx 7 \times 10^{-5}$, corresponding to a diurnal tide amplitude of $130 \mathrm{~m}$. Future accurate measurements of the tidal amplitude at the core-mantle boundary and of the libration amplitude should confirm our prediction.

In the optimal case for instability, Fig. 5 shows that the liquid core of Europa is unstable, as already suggested by Kerswell \& Malkus (1998), even when taking the Joule dissipation due to the presence of Jupiter magnetic field into account. The typical growth time of the instability to be around 400 years, and the associated dissipation to be on the order of $P \sim 10^{10} \mathrm{~W}$. This corresponds to $Q^{*} \sim 10^{7}$, and is two orders of magnitude below the conservative estimation of $3 \times 10^{12} W$ for the tidal heating rate on Europa (O'Brien et al. 2002). In reality, the silicate mantle of Europa should behave more rigidly. Since the libration amplitude of the mantle and the amplitude of the tidal distortion at the core-mantle boundary are not known yet, all intermediate behaviors are explored in Fig. 7a. We conclude that high libration amplitude and/or rather large elliptical distortion are needed for Europa's core to be unstable; nevertheless, the parameter range for instability is rather wide in Fig. 7a and seems to be reachable: we thus expect the core of Europa to be unstable. Results for Europa subsurface ocean are shown in Fig. 7b. The elastic behavior of the icy crust above the subsurface ocean is expected to behave in the deformable limit $(\mathcal{1} \ll$ ), which correspond to parameters close to the upper right hand corner. As also shown in Fig. 5, Europa's ocean is therefore unstable.

Concerning the two last Galilean moons, the core of Ganymede and the subsurface oceans of Callisto and Ganymede are found to be stable in the optimal case (Fig. 5). An LDEI is 


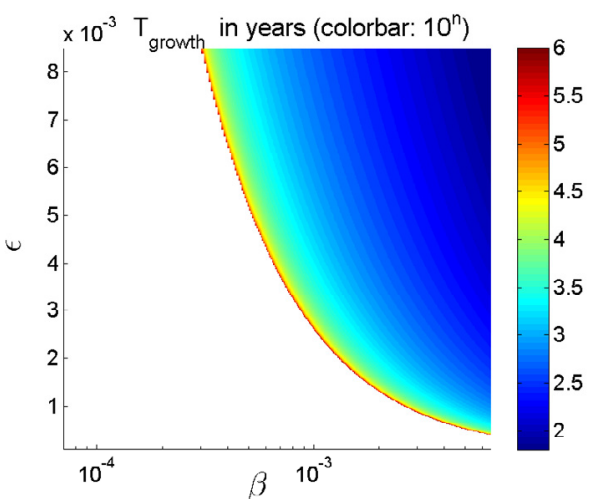

Fig. 6. Evolution of the typical growth time $T_{\text {growth }}=1 / \sigma$ of the instability in Io with the tidal bulge ellipticity $\beta$ and the libration amplitude $\epsilon$ ( $\alpha=2.62$ ). The upper right corner corresponds to the optimal case considered in Kerswell \& Malkus (1998) and Herreman et al. (2009), the upper left corner corresponds to optical librations with a small tidal amplitude corresponding to diurnal tides $\left(\beta=7 \times 10^{-5}, \epsilon=2 e=0.0082\right)$ and the lower right corner corresponds to the region of physical librations of the static bulge $\left(\beta=0.006, \epsilon=1.3 \times 10^{-4}\right)$. The white zone corresponds to the stable zone where the instability cannot grow because of dissipative effects (at the boundary with the colored zone, the growth time is infinite). The colorbar range is chosen so that color variations are visible.

improbable today. Figure 5 also shows that the subsurface ocean of Titan is probably stable, because even in the optimal case for the LDEI, it remains in the vicinity of the threshold.

\subsubsection{Super-Earths}

The recent discovery of extrasolar telluric planets gives typical examples of synchronized planets in close orbit around their host stars. This particular astrophysical configuration should lead to a vigorous LDEI in their possible liquid cores. In this section, we consider three Super-Earths, expected to be telluric: $55 \mathrm{CnC}$ e, CoRoT-7b, and GJ 1214b, at $D=0.0156 \mathrm{AU}, D=0.0172 \mathrm{AU}$, and $D=0.0143 \mathrm{AU}$ from their host star respectively. The data used here are from Winn et al. (2011) for $55 \mathrm{CnC}$ e, Valencia et al. (2010) for CoroT-7b, and Charbonneau et al. (2009) for GJ 1214b. They are given in Table 7. For CoRoT-7b, the work of Léger et al. (2011) predicts a core composed of liquid metal, representing $11 \%$ of the total planetary volume, as for the actual Earth. The presence of a liquid core in Super-Earths is still not clear (e.g. Wagner et al. 2011, for CoroT-7b), but we can reasonably assume that a planetary liquid core could occupy about one third of the planet radius, which corresponds to $4 \%$ of the total planetary volume. Because of the proximity of the parent star, these extrasolar planets are expected to be synchronized. The actual orbital eccentricities of CoRoT-7b and GJ 1214b are not known. If they are fully circularized and synchronized, no elliptical instability can grow. In contrast, if a small libration exists, previous stability formula can be used. We assume here an orbital eccentricity of $e=0.001$, well beyond the detection limit. Figure 5 shows that in the optimal case these three Super-Earths cores are clearly unstable with a good level of confidence, which means that the LDEI is probably present in their liquid layers. One can see that this result is not very sensitive to the hypothesis on the size of the considered liquid core: for instance, with the values of Table 7, CoroT-7b becomes stable for a liquid core aspect ratio $R_{2} / R$ below $1 \%$ (for the optimal case i.e. optical

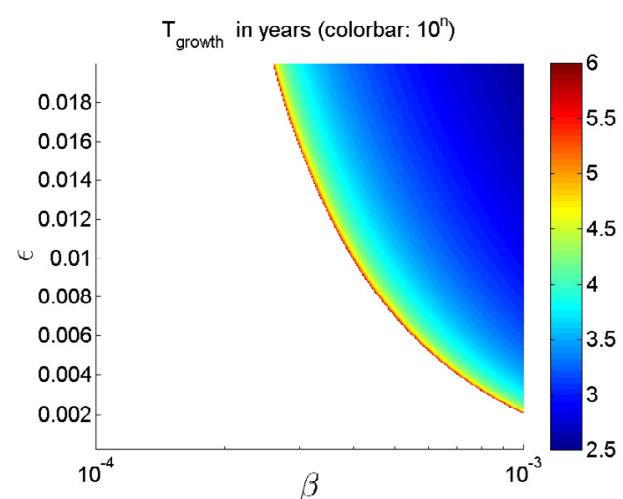

(a)

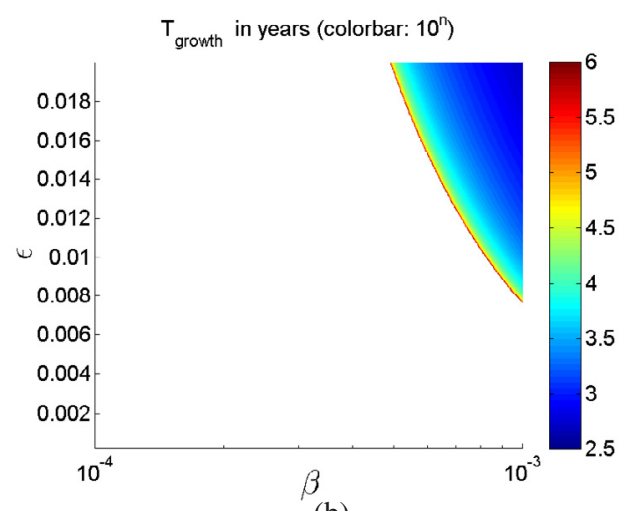

(b)

Fig. 7. Same as Fig. 6 but for Europa, considering a) its possible liquid core and b) a $100 \mathrm{~km}$ depth subsurface ocean. Both the core and the subsurface ocean of Europa are expected to behave in the deformable limit, locating their states in the upper right corner of the diagram, so both are found to be unstable.

librations and equilibrium tides). In these estimates, we use the hydrostatic tidal deformation, which underestimates the real tidal deformation. Also, higher orbital eccentricity would lead to more unstable configurations. Figure 8 shows the influence of these uncertainties, as well as the effect of smaller libration amplitudes and tidal deformations. The proximity of the known Super-Earths with their host stars leads to strong tidal deformations, and the LDEI is then able to grow from very small libration amplitudes, as shown in Fig. 8 (the true tidal deformation, greater than the hydrostatic value, leads to an LDEI for a wide range of libration amplitudes). Same conclusions are obtained for Kepler-10b (Batalha et al. 2011), assuming the same hypothesis. To conclude, the presence of the LDEI in their liquid cores is very probable, regardless of the uncertainty ranges.

\section{Conclusion and discussion}

In conclusion, we have investigated theoretically the elliptical instability in telluric celestial bodies. New analytical results were determined to fill the gap between previous studies and astrophysical applications. In particular, we have derived generic formulas for the growth rate of the elliptical instability driven respectively by tides in non-synchronized bodies (TDEI) and libration in synchronized ones (LDEI), in the presence of imposed magnetic and thermal base fields. It was shown that an elliptical instability is strongly expected in the core of Europa, $55 \mathrm{CnC}$ e, CoRoT-7b, and GJ 1214b, as well as in the subsurface ocean of Europa. Those results are valid for the present state of the considered bodies and do not preclude any elliptical instability 
Table 7. Physical and orbital characteristics used for the stability calculations in exoplanets.

\begin{tabular}{lccc}
\hline \hline & CoRoT-7b & GJ 1214b & 55 CnC e \\
\hline$M$ (in Earth's mass) & 4.8 & 6.55 & 8.57 \\
$R$ (in Earth's radius) & 1.68 & 2.678 & 1.63 \\
$T_{\text {orb }}(d)$ & 0.854 & 1.58 & 0.7365 \\
$R_{2} / R$ & $1 / 3^{a}$ & $1 / 3^{b}$ & $1 / 3^{c}$ \\
$e$ & $0.001^{c}$ & $0.001^{c}$ & $0.057^{d}$ \\
$\eta$ & 0 & 0 & 0 \\
$E\left(\times 10^{16}\right)$ & 9.4 & 6.8 & 8.6 \\
$\beta\left(\times 10^{3}\right)$ & 7 & 6 & 5 \\
$\sigma\left(\mathrm{yr}^{-1}\right)$ & 0.01 & 0.005 & 0.45 \\
\hline
\end{tabular}

Notes. We take as typical value $v=10^{-6} \mathrm{~m}^{2} \mathrm{~s}^{-1}$, consistent with a $\mathrm{Fe} / \mathrm{Fe}-\mathrm{S}$ composition. We use the formula (4) to estimate the tidal bulge ellipticity. ${ }^{(a)}$ Coherent with Léger et al. (2011). ${ }^{(b)}$ Coherent with the values from Nettelmann et al. (2011). ${ }^{(c)}$ Hypothesis assumed. ${ }^{(d)}$ Winn et al. (2011).

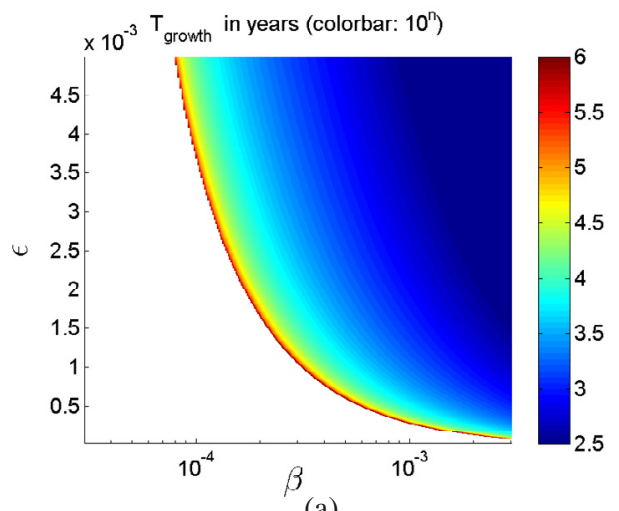

(a)

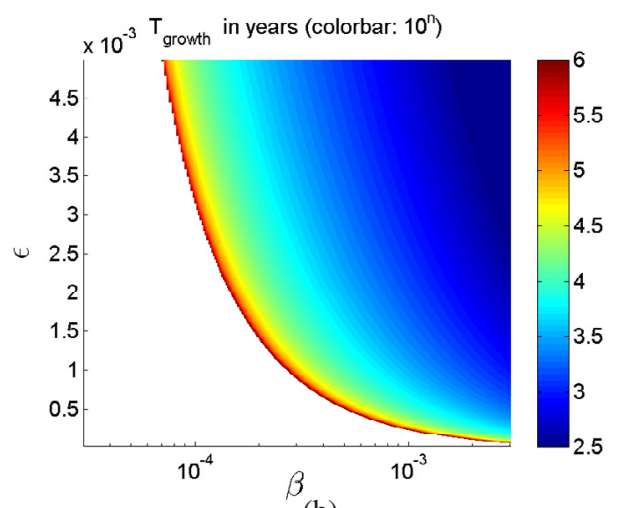

(b)

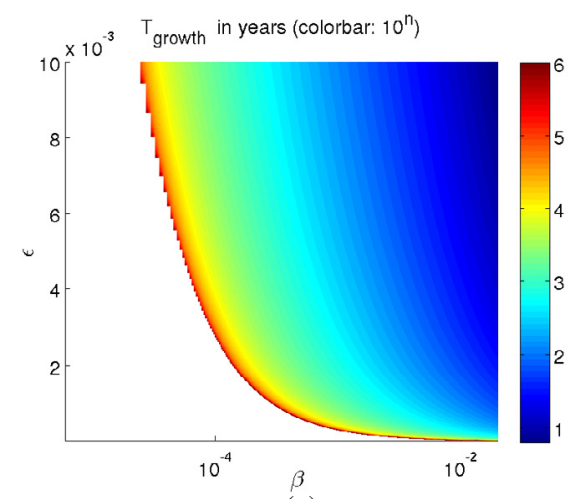

(c)

Fig. 8. Same as Fig. 6 but for CoroT-7b in a), GJ 1214 b in b), and 55 $\mathrm{CnC}$ e in c). in the past. For instance, the early Earth core was clearly unstable, because of the larger gravitational distortions when the Moon was closer.

One can wonder about the signatures and consequences of such an instability on the planetary dynamics. A first consequence would be on the orbital evolution and synchronization process: indeed, the elliptical instability generates threedimensional turbulent flows with cycles of growth, saturation, fluctuations, and relaminarization (e.g. Le Bars et al. 2010). Timescales involved range typically between the spin period and the growth time of the instability. Dissipation rates on the planetary scale, and consequently the orbital evolution, may then follow the same variations, with periods of rapid evolution when an elliptical instability is present, followed by more quiescent periods, for instance when the forbidden zone is reached. This increased dissipation should accelerate the synchronization process, as described in Le Bars et al. (2010), and this range of timescales should appear in the evolution of the spin rotation rate.

The second consequence would be on heat flux variations at the planetary surface. Indeed, as shown in Cébron et al. (2010c); Lavorel \& Le Bars (2010), flows driven by elliptical instability are very efficient in transporting heat by advection. As a result, subadiabatic cores should not be regarded as thermal blankets in the presence of the elliptical instability. The usual Nusselt number $N u=Q_{\text {tot }} / Q_{\text {diff }}$, the ratio between the total outward heat flux $Q_{\text {tot }}$ and the purely diffusive outward heat flux $Q_{\text {diff }}$, which is associated to this heat advection, is given by the following scaling law, verified both experimentally (Lavorel \& Le Bars 2010) and numerically (Cébron et al. 2010c):

$N u=\frac{0.01}{\sqrt{E}}$

This leads to a total outward heat flux advected by the elliptical instability about $N u \approx 3 \times 10^{4}$ times greater than a purely diffusive outward heat flux. Besides, in the presence of natural thermal convection, the superimposition of chaotic elliptically driven flows would induce large-scale variations in the same amplitude.

Finally, internal flows driven by elliptical instability are directly responsible for magnetic field generation. The question of whether LDEI and TDEI are dynamos-capable is still open and remains out of the reach of the currently available numerical capacity, but elliptically driven flows induce a magnetic field from an existing background one. To estimate a typical amplitude of such an induced field, we can use the results of our WKB approach. This shows that the dimensionless induced magnetic field inside the core and the instability velocity $\boldsymbol{u}_{0}$ are systematically related by

$\boldsymbol{B}=\mathrm{i} \frac{R m k_{x_{3}}}{k^{2}} \boldsymbol{u}_{0}$,

where $k$ and $k_{x_{3}}$ are the norm and the axial component, respectively, of the wave vector of the excited mode of the elliptical instability. This generic expression shows that the induced magnetic field due to the elliptical instability is systematically proportional to and in quadrature with the velocity field due to the instability. For the TDEI, $k_{x_{3}}=k / 2$, and for the LDEI, $k_{x_{3}}=\omega_{0} k / 4$ (see respectively Appendices A and B). Then, assuming that at saturation, the typical flow induced by instability is commensurate with the differential rotation between the fluid and the elliptical distortion (see Table 1), we estimate the 
surface field by

$b_{\text {surf }}=\frac{R m k_{x_{3}}}{k^{2}} \frac{\Delta \Omega}{\Omega}\left(\frac{R_{2}}{R}\right)^{3}$,

where $R$ is the planet radius. Starting from the Jovian magnetic field component along the rotation axis, the LDEI in Europa subsurface ocean is capable of inducing surface variations of up to $\sim 0.1 \%$ of the ambient field (reached for $k=2 \pi /(1-\eta)$ and optical librations, i.e. $\epsilon=0.0188)$, and LDEI in its core up to $\sim 100 \%$ of the ambient field at the surface (reached for $k=2 \pi /(1-\eta)$ and physical librations, i.e. $\left.\epsilon=2 \times 10^{-4}\right)$. Considering Galileo's E4 flyby of Europa (see Zimmer et al. 2000; Kabin et al. 1999), the background $z$-component of the magnetic field is modified from $410 \mathrm{nT}$ to $380 \mathrm{nT}$ at a distance of Europa about 1.5 Europa radius. This modification of $30 \mathrm{nT}$ is on the same order of magnitude as the possibly LDEI induced magnetic field from the core. Internal sources should thus be considered in addition to plasma currents (Kabin et al. 1999) for interpretating Europa's magnetic signal.

To finish with, one should notice that the TDEI and LDEI studied here for telluric planets, can also affect the giant gaseous planets of our solar system (Wicht \& Tilgner 2010), as well as extrasolar gaseous planets such as the hot-Jupiters, whose dramatic tidal deformations should excite vigorous elliptical instabilities in the planetary atmospheres, but also in their host stars (Rieutord 2003; Ou et al. 2007; Cébron et al. 2011).

Acknowledgements. The authors acknowledge S. Le Dizès for his valuable help on the stability analysis; J. Noir and A. Sauret for fruitful discussions on libration; J. Besserer and G. Tobie for fruitful discussions and values on tidal heating in the Galilean moons and Titan.

\section{Appendix A: Stability analysis for non-synchronized systems}

Solving (16), we find the trajectories

$\boldsymbol{x}=r\left[\begin{array}{c}\sqrt{1+\beta} \cos \left(t \sqrt{1-\beta^{2}}\right) \\ \sqrt{1-\beta} \sin \left(t \sqrt{1-\beta^{2}}\right) \\ 0\end{array}\right]$

where without loss of generality, the origin of time has been defined such that the initial position along the trajectory is $\boldsymbol{x}_{(t=0)}=$ $\left[x_{1}, x_{2}\right]=[r \sqrt{1+\beta}, 0]$ (the results of the WKB analysis do not depend on this chosen initial position).

The solution to Eq. (22) at order 1 in $\beta$ along a streamline (A.1) writes as

$\boldsymbol{k}=\left[\begin{array}{l}k_{x_{1}} \\ k_{x_{2}} \\ k_{x_{3}}\end{array}\right]=\left[\begin{array}{cccc}k_{x_{10}} & \cos t-k_{x_{20}} & \sin t+\beta k_{x_{20}} & \sin t \\ k_{x_{10}} & \sin t+k_{x_{20}} \cos t+\beta k_{x_{10}} & \sin t \\ k_{0} \cos a & \end{array}\right]$

where $k_{x_{10}}, k_{x_{20}}$, and $k_{0}$ are constant, and $a$ is the angle between the wave vector and the rotation axis. We define the phase $\phi$ of the wave vector by writing $k_{x_{10}}=k_{0} \sin a \cos \phi$, $k_{x_{20}}=k_{0} \sin a \sin \phi$.

We are now in a position to solve the system of linearized equations given in Sect. 3.3. To do so, we use as unknowns the vertical velocity of the perturbed field $u_{3}$ and its vertical vorticity $W_{3}=\partial_{x_{1}} u_{2}-\partial_{x_{2}} u_{1}=\mathrm{i}\left(k_{x_{1}} u_{2}-k_{x_{2}} u_{1}\right)$, as well as the vertical component $b_{3}$ of the perturbed magnetic field and the corresponding magnetic vertical vorticity $C_{3}=\mathrm{i}\left(k_{x_{1}} b_{2}-k_{x_{2}} b_{1}\right)$. The resolution is then straightforward (see Herreman et al. 2009).

At order 0 in $\beta$, the system reduces to an harmonic equation for $u_{3}$, giving a dispersion relation with a pulsation $f=$
$2\left(1+\Omega^{G}\right) \cos a$, with the quantity $\Omega^{G}=\Omega_{\text {orb }} /\left(\Omega_{\text {spin }}-\Omega_{\text {orb }}\right)$ already used by Kerswell (2002); Le Bars et al. (2010). Solvability conditions imply non-trivial solutions only if $f=1$, which gives the resonance condition $\cos a=1 /\left(2\left(1+\Omega^{G}\right)\right) \in[-1,1]$. This means that the instability cannot grow when $\Omega / \Omega_{\text {orb }} \in[-1 ; 1 / 3]$, which is the so-called forbidden zone. Outside this band, the growth rate is determined by the nullity of the determinant of the solvability condition system. It is then maximized over all values of wave vector phase $\phi$. The maximum is obtained for $\phi=\pi / 4$ and the inviscid growth rate writes as

$$
\begin{aligned}
\sigma_{\text {inv }}= & \frac{\left(2 \Omega^{G}+3\right)^{2}}{16\left|1+\Omega^{G}\right|^{3}} \sqrt{\beta^{2}-4 \zeta^{2}}-\frac{\Lambda}{4\left|1+\Omega^{G}\right|^{3}\left(1+R m^{2} k^{-4}\right)} \\
& -\frac{k^{2} \tilde{\operatorname{Ra}} r \partial_{r} \theta}{8\left(1+k^{4} E^{2} / \operatorname{Pr}^{2}\right)\left|1+\Omega^{G}\right|^{3}}
\end{aligned}
$$

with

$\zeta=\frac{\left(k^{4}+R m^{2}\right) \tilde{\operatorname{Ra}} r \partial_{r} \theta-2 k^{2} R m \Lambda\left(1+k^{4} E^{2} / \operatorname{Pr}^{2}\right)}{\left(2 \Omega^{G}+3\right)^{2}\left(1+k^{4} E^{2} / \operatorname{Pr}^{2}\right)\left(k^{4}+R m^{2}\right)}$,

where $r$ and $\partial_{r} \Theta$ are the radius and the dimensionless radial gradient of temperature base field on the considered streamline, respectively. In the absence of viscous boundary damping (discussed in Sect. 3.6), the inviscid growth rate is a correct approximation of the viscous growth rate when the viscous diffusion term $-k^{2} E$ in Eq. (19) is negligible, i.e. for perturbations of wavelength greater than the Ekman thickness $\sqrt{E}$. Equation (A.3) takes the thermal and magnetic diffusions into account. In a typical liquid core, the thermal Prandtl number is about $O(0.1-1)$. Neglecting the viscous diffusion term also leads to neglecting the thermal diffusion term $-k^{2} E / P r$ of Eq. (19). But since the magnetic Prandtl number is about $P m \approx 10^{-6}$ (e.g. Christensen \& Aubert 2006), the magnetic diffusion term $-k^{2} / R m=-k^{2} E / P m$ of Eq. (21) appears as the dominant diffusion mechanism. It can nevertheless be neglected within the limit of large wavenumbers, where Eq. (A.3) gives (24).

\section{Appendix B: Stability analysis for synchronized systems}

We consider that the differential rotation between the fluid and the elliptical distortion writes as $1-\gamma_{(t)}=\epsilon \cos \left(\omega_{0} t+q\right)$. The phase $\mathrm{q}$ is introduced here because we define the origin of time such that the initial position of the considered trajectory is $x_{2}=0$. Equation (16) can be solved analytically to find the trajectories. In the particular case $q=0$, the solution can be written in the following compact form:

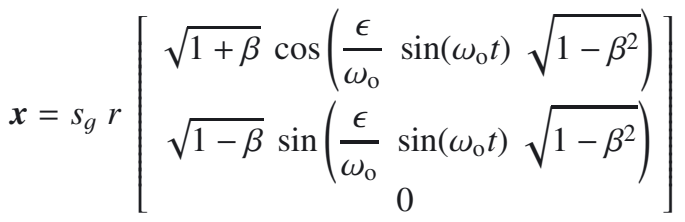

with $s_{g}=\operatorname{sgn}\left(\cos \left(\omega_{0} t\right)\right)$. In the general case, the wave vector associated to this flow writes as

$\boldsymbol{k}=\left[\begin{array}{l}k_{x_{1}} \\ k_{x_{2}} \\ k_{x_{3}}\end{array}\right]=\left[\begin{array}{c}k_{x_{10}}-\frac{k_{x_{20}}}{M}\left(\sin \left(\omega_{\mathrm{o}} t+q\right)-\sin q\right)(1-\beta) \epsilon \\ k_{x_{20}}+\frac{k_{x_{10}}}{M}\left(\sin \left(\omega_{0} t+q\right)-\sin q\right)(1+\beta) \epsilon \\ k_{0} \cos a\end{array}\right]$ 
where $k_{x_{10}}, k_{x_{20}}$, and $k_{0}$ are constant and $a$ is the angle between the wave vector and the rotation axis. We define again the phase $\phi$ by $k_{x_{10}}=k_{\mathrm{o}} \sin a \cos \phi, k_{x_{20}}=k_{\mathrm{o}} \sin a \sin \phi$.

At leading order in $\epsilon \beta$, the dispersion relation gives $f=$ $2 \cos a$, and the solvability conditions system admits non-trivial solutions for $f=\omega_{0} / 2$. Consequently, the authorized band is given by $\cos a=\omega_{\mathrm{o}} / 4 \in[-1,1]$ i.e. $\left|\omega_{\mathrm{o}}\right| \leq 4$. The growth rate is determined at order 1 in $\epsilon \beta$ and must be maximized above the phases $q$ and $\phi$. The maximum is reached for $q=0, \phi=\pi / 4$ and gives in the absence of thermal and magnetic fields

$$
\frac{\sigma_{\text {inv }}}{\epsilon \beta}=\frac{16+\omega_{\mathrm{o}}^{2}}{64}
$$

Kerswell \& Malkus (1998) have performed a global approach to the same instability, explicitly considering inertial waves coupling in a spheroidal geometry. In the absence of magnetic and thermal fields, they found a maximum inviscid growth rate $\sigma_{\text {inv }} /(\epsilon \beta)=25 / 128$, very close to our value $\sigma_{\text {inv }} /(\epsilon \beta)=17 / 64$. The small difference between the two values is due to the influence of the spheroidal geometry considered in Kerswell \& Malkus (1998), leading to more restrictive conditions for destabilization than our local analysis. Similarly, for purely hydrodynamic flow with a stationary deformation as studied in Sect. 3.4, our analysis gives the inviscid growth rate $\sigma_{\text {inv }} / \beta=9 / 16$, whereas a global analysis with inertial waves of a spheroid leads to the slightly lower value $\sigma_{\text {inv }} / \beta=1 / 2$ (see Lacaze et al. 2004). Taking the uncertainties on the different parameters for planetary application into account, these small differences can be disregarded and the local approach can be used confidently, which presents the strong advantage of providing an explicit formula for the growth rate.

Taking a buoyancy of order $\epsilon \beta$ into account, as well as the induction equation and a Lorentz force on the order of $\epsilon \beta$ in the presence of an imposed vertical magnetic field $B_{0}$, we obtain the growth rate

$$
\begin{aligned}
\sigma_{\text {inv }}= & \frac{16+\omega_{\mathrm{o}}^{2}}{64} \sqrt{(\epsilon \beta)^{2}-4 \omega_{\mathrm{o}}^{2} \zeta_{2}^{2}} \\
& -\frac{\omega_{\mathrm{o}}^{2} \Lambda}{16\left(1+k^{-4} \omega_{\mathrm{o}}^{2} R m^{2} / 4\right)}-\frac{\left(16+\omega_{\mathrm{o}}^{2}\right) k^{2} \tilde{R a} r \partial_{r} \theta}{16\left(\omega_{\mathrm{o}}^{2}+4 k^{4} E^{2} / \operatorname{Pr}^{2}\right)}(\mathrm{B} .
\end{aligned}
$$

with

$$
\zeta_{2}=\frac{F}{\left(16+\omega_{\mathrm{o}}^{2}\right)\left(4 k^{4}+\omega_{\mathrm{o}}^{2} R m^{2}\right)\left(\omega_{\mathrm{o}}^{2}+4 k^{4} E^{2} / \operatorname{Pr}^{2}\right)}
$$

where $F=\left(64+\omega_{\mathrm{o}}^{2}\left(4 k^{4}+\operatorname{Rm}^{2}\left(16+\omega_{\mathrm{o}}^{2}\right)\right) \tilde{R a} r \partial_{r} \theta-\right.$ $4 k^{2} \omega_{\mathrm{o}}^{2} R m \Lambda\left(\omega_{\mathrm{o}}^{2}+4 k^{4} E^{2} / P r^{2}\right)$, and where $r$ and $\partial_{r} \theta$ are respectively the radius and the dimensionless temperature radial gradient of the considered streamline. As discussed in Appendix A, in astrophysical applications, the thermal diffusion can be neglected $(E / P r=0)$. In this case, Eq. (B.4) gives (25) in the limit of large wavenumbers.

\section{Appendix C: Is the diurnal tide stabilizing or destabilizing for the elliptical instability?}

In this work, the periodic forcing due to diurnal tides has been neglected for synchronized bodies. It is thus legitimate to calculate its influence on the LDEI growth. To answer this question, we consider the simplest but severe case of a body with a non rotating (i.e. $\gamma=0$ ) diurnal tide of amplitude $\beta_{(t)}=\beta_{1} \cos (M t+q)$, where $\beta_{1}=3 e \beta$ and there is no global rotation. Then, the base flow (11) reduces to

$$
\begin{aligned}
\boldsymbol{U}=\left[-\left(1+\beta_{1} \cos (M t+q)\right) x_{2} \boldsymbol{e}_{\mathrm{x}_{1}}\right. \\
\\
\left.+\left(1-\beta_{1} \cos (M t+q)\right) x_{1} \boldsymbol{e}_{\mathrm{x}_{2}}\right] .
\end{aligned}
$$

Once again, the phase $q$ is introduced here because we fix the phase of streamlines. The streamlines are not known analytically. The dispersion relation gives the pulsation $f=2 \cos a$, and the solvability conditions give resonances for $f=(2+M) / 2$ and $f=(2-M) / 2$. The authorized band is thus $|M| \leq 6$. In the limit of low $M$, the maximal growth rate is obtained with $f=(2-M) / 2$ for $\phi=-\pi / 4$ and $q=0$ :

$\sigma_{\text {inv }}=\left[\frac{9}{16}+\frac{81}{64} M+\left(\frac{1}{8}-\frac{15 \pi^{2}}{32}\right) M^{2}\right] \beta_{1}$

at the order $O\left(\Lambda^{2}\right)+O\left(\beta_{1} M^{3}\right)+O\left(\beta_{1}{ }^{2}\right)$. This expression agrees with equation (24) for $\Lambda=0$ and $\tilde{R} a=0$, in the limit $M=0$. The expression (C.2) shows that slow oscillations of the amplitude of the tidal bulge are not inhibiting for the elliptical instability. On the contrary, the growth rate is enhanced compared to the case of constant amplitude for low $M$, which means that the diurnal tide would be destabilizing in this case. In the case of planetary interest with $M=1$, the maximum growth rate writes as

$\sigma_{\text {inv }}=\frac{25}{128} \beta_{1}$

which again shows that the diurnal tide can drive an elliptical instability. This effect will thus be superimposed on the TDEI and LDEI mechanisms already studied, but with a slower growth rate, since $\beta_{1} \ll \beta$.

\section{Appendix D: Resonances of gravito-inertial waves}

To clarify the influence of a thermal field with a buoyancy force that is on the order of zero in $\beta$, we consider the generic case with the elliptical gravitational iso-potentials of ellipticity $n \beta$ and elliptical base-field isotherms of ellipticity $m \beta$, where $n$ and $m$ are arbitrary constants. This generic notation is needed to deal with all cases, as studied for instance in Le Bars \& Le Dizès (2006), Lavorel \& Le Bars (2010) and Cébron et al. (2010c). In focusing on dynamic tides in a non-synchronized system, one would expect the isotherms to follow the streamlines (because of the small thermal diffusion coefficient), as well as the iso-potentials. Hence, $n=m=1$. In contrast, looking at the elliptical instability in a subsurface ocean underlain by a rigid mantle, one would expect the iso-potentials to remain quasi-circular $(n=0)$. Besides this, in the presence of a static bulge, one would expect the system to naturally return to a configuration with $m=0$ by the generation of baroclinic motions. All situations with $0 \leq m, n \leq 1$ are possible, and one can even imagine other azimuthal periodicities, for instance those due to local variations of temperature.

In the case of the TDEI, the WKB approach including zeroth-order buoyancy forces in $\beta$ is tractable. Unlike the cases studied in Appendices A and B, the forbidden band where the instability does not exist is now modified by the thermal field and is given by $f_{0} \leq 1$, where $f_{0}=\sqrt{4\left|1+\Omega^{G}\right|^{2}+\tilde{R} a r \partial_{r} \Theta}$. Outside this band, the inviscid growth rate is given, in the limit of large wavenumbers, by:

$$
\begin{aligned}
\frac{\sigma_{\text {inv }}}{\beta}= & \frac{\left(2 \Omega^{G}+3\right)^{2}+\left[1+2\left(1+\Omega^{G}\right)(m-n)-n\right] \tilde{\operatorname{Ra}} r \partial_{r} \Theta}{16\left|1+\Omega^{G}\right|^{3}+4 \tilde{R a}\left|1+\Omega^{G}\right| r \partial_{r} \Theta} \\
& -\frac{\Lambda}{4\left|1+\Omega^{G}\right|^{3}} \frac{1}{\beta}
\end{aligned}
$$


We can compare the role of the temperature field in (24) and (D.1). In expression (24), the temperature field acts as a simple supplementary stabilizing term that corrects the inviscid purely hydrodynamic growth rate. But in the derivation of Eq. (D.1), waves and resonances (as well as the forbidden band) are modified by buoyancy forces, leading to a modification of the prefactor of $\beta$. Actually, the elliptical instability now results from resonances of gravito-inertial waves and should be called the gravito-elliptical instability (see Le Bars \& Le Dizès 2006; Guimbard et al. 2010). As shown below, the supplementary resonances associated to gravito-inertial waves allow the temperature to be destabilizing in certain cases. The same conclusions can be obtained for the magnetic field when Lorentz forces are taken into account at zeroth order in $\beta$ in the limit of ideal magnetohydrodynamic: the elliptical instability then results from resonances between magneto-inertial waves (Kerswell 1993a, 1994); the forbidden band is modified by the magnetic field; and as shown by Lebovitz \& Zweibel (2004); and the magnetic field can be either stabilizing or destabilizing depending on the case being considered (see also Herreman 2009; Mizerski \& Bajer 2009, 2011). Naturally, these conclusions are also valid for the LDEI.

In Le Bars \& Le Dizès (2006) and in the experiments of Lavorel \& Le Bars (2010), the TDEI is studied for a stationary bulge $\left(\Omega^{G}=0\right)$ with circular iso-potentials and elliptical isotherms $(n=0, m=1)$, and the considered temperature profile gives $r \partial_{r} \Theta=-1$. In this particular case, in the absence of magnetic field, Eq. (D.1) recovers their result:

$\sigma_{\text {inv }}=\frac{9-3 \tilde{R a}}{16-4 \tilde{R a}} \beta$.

As already noticed by Le Bars \& Le Dizès (2006), a thermal stable stratification $(\tilde{R a}<0)$ is then destabilizing for the elliptical instability, but, in constrast, the temperature field stabilizes the instability for $n=m=1$. This high sensitivity of the growth rate of the elliptical instability to the specific gravitational and thermal fields is confirmed by numerical simulations. Using the method described in Cébron et al. (2010c), we consider the simple case $\Omega^{G}=0$ and $K=0$, the temperature field being established by a temperature contrast between the two boundaries. When $n=m=1$, the growth rate (D.1) is enhanced when $\tilde{R a}$ is increased. As shown in Fig. D.1, this is in perfect agreement with the numerical simulations in a cylindrical shell. In the experimental setup of Lavorel \& Le Bars (2010), the gravity is replaced by the centrifugal acceleration, as in Carrigan \& Busse (1983), and the associated equipotentials are circular, i.e. $n=0$ and $m=0$, as shown in Fig. D.1. In this case an increasing $\tilde{R a}$ indeed leads to a lower growth rate and the numerical simulations agree with the predicted growth rate.

The conclusions for an autogravitating ellipsoidal shell are more complex. Cébron et al. (2010c) find that an increasing $\tilde{R a}$ leads to a lower growth rate, which contradicts the prediction of the theoretical growth rate. This difference comes from the fact that the thermal stratification propagates the influence of the boundary inside the bulk: the WKB analysis, based on local stability, cannot handle this feature. In the spherical geometry, we can, however, notice that changes induced by $\tilde{R a}$ are small for $-1 \leq \tilde{R a}<1$ and remain close to the estimates for an autogravitating cylinder.

Those results clearly illustrate the high sensitivity of the growth rate of the elliptical instability to the specific gravitational and thermal fields, as well as to the considered geometry. In planetary applications, stratification (i.e. $\tilde{R} a<0)$ generally leads to stabilization, as in the limit of small $\tilde{R} a$ presented in the

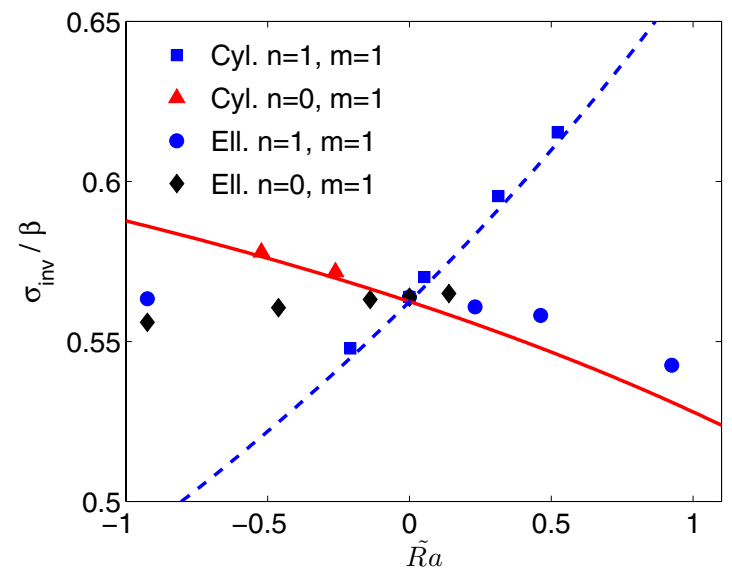

Fig. D.1. Growth rate of the TDEI for a cylindrical shell of aspect ratio $H / R=2$ with an elliptical cross section $(\eta=0.2, E=0.0036, \beta=$ $0.47, \operatorname{Pr}=1)$ and an ellipsoidal shell $(\eta=0.3, E=0.0029, \beta=$ $0.317, \operatorname{Pr}=1)$ with a rotation axis of length $c=(a+b) / 2$. The figure compares the numerical growth rate in the autogravitating case where the gravity is given by the Poisson equation for the gravitational potential of a homogeneous fluid (see Cébron et al. 2010c, for details), and the case where the gravity is played by a centrifugal force $(n=0)$, as in the experiments of Lavorel \& Le Bars (2010). The numerical growth rate is translated vertically in the figure, to match the inviscid growth rate $9 / 16$ at $\tilde{R a}=0$, which corresponds to a surfacic damping term coefficient $\alpha=3.24$ for the cylindrical shell (squares and triangles) and $\alpha=3.1$ for the ellipsoidal shell (circles and diamonds). Theoretical growth rates are shown by a continuous red line for $n=0, m=1$ and a dashed blue line for $n=m=1$.

main text; however, in this case, stratification can only stabilize elliptical instability when $\tilde{R a}=O(1-10)$, which is never the case.

\section{References}

Aldridge, K., \& Toomre, A. 1969, J. Fluid Mech., 37, 307

Aldridge, K., Seyed-Mahmoud, B., Henderson, G., \& van Wijngaarden, W. 1997, Phys. Earth Planet. Int., 103, 365

Anderson, B., Acuña, M., Korth, H., et al. 2010, Space Sci. Rev., 152, 307

Arkani-Hamed, J. 2009, Icarus, 201, 31

Arkani-Hamed, J., Seyed-Mahmoud, B., Aldridge, K., \& Baker, R. 2008, J. Geophys. Res., 113, E06003

Baland, R., \& Van Hoolst, T. 2010, Icarus, 209, 651

Bars, M. L., Wieczorek, M., Karatekin, Ö., Cébron, D., \& Laneuville, M. 2011, Nature, 479, 215

Batalha, N., Borucki, W., Bryson, S., et al. 2011, ApJ, 729, 27

Bayly, B. 1986, Phys. Rev. Lett., 57, 2160

Benest, D. 1990, Modern methods in celestial mechanics (Éditions Frontières)

Bland, M., Showman, A., \& Tobie, G. 2008, Icarus, 198, 384

Bland, M., Showman, A., \& Tobie, G. 2009, Icarus, 200, 207

Busse, F. 1968, J. Fluid Mech., 33, 739

Busse, F. 2010, J. Fluid Mech, 650, 505

Calkins, M., Noir, J., Eldredge, J., \& Aurnou, J. 2010, Phys. Fluids, 22, 086602

Carrigan, C., \& Busse, F. 1983, J. Fluid Mech., 126, 287

Cébron, D., Bars, M. L., Leontini, J., Maubert, P., \& Gal, P. L. 2010a, Phys. Earth Planet. Int., 182, 119

Cébron, D., Le Bars, M., \& Meunier, P. 2010b, Phys. Fluids, 22, 116601

Cébron, D., Maubert, P., \& Le Bars, M. 2010c, Geophys. J. Int., 182, 1311

Cébron, D., Moutou, C., Le Bars, M., Le Gal, P., \& Farès, R. 2011, EPJ Web of Conferences, 11, ed F. Bouchy, R. Díaz, \& C. Moutou

Chan, K. H., Liao, X., \& Zhang, K. 2011a, Phys. Earth Planet. Int., 187, 391

Chan, K. H., Liao, X., \& Zhang, K. 2011b, Phys. Earth Planet. Int., 187, 404

Charbonneau, D., Berta, Z., Irwin, J., et al. 2009, Nature, 462, 891

Christensen, U., \& Aubert, J. 2006, Geophys. J. Int., 166, 97

Craik, A. 1989, J. Fluid Mech., 198, 275

Craik, A., \& Criminale, W. 1986, Proc. Roy. Soc. London, Ser. A, Math. Phys. Sci., 406, 13

Friedlander, S., \& Vishik, M. 1991, Phys. Rev. Lett., 66, 2204 
Garrick-Bethell, I., Wisdom, J., \& Zuber, M. 2006, Science, 313, 652

Goldreich, P., \& Mitchell, J. 2010, Icarus, 209, 631

Greenberg, R. 2009, ApJ, 698, L42

Greenberg, R., Hoppa, G., Bart, G., \& Hurford, T. 2003, Cel. Mech. Dyn. Astron., 87, 171

Greenspan, H. 1968, The theory of rotating fluids (Cambridge University Press) Greff-Lefftz, M., Metivier, L., \& Legros, H. 2005, Cel. Mech. Dyn. Astron., 93, 113

Guimbard, D., Le Dizès, S., Le Bars, M., Le Gal, P., \& Leblanc, S. 2010, J. Fluid Mech., 660, 240

Hand, K., \& Chyba, C. 2007, Icarus, 189, 424

Herreman, W. 2009, Ph.D. Thesis, Université de Provence - Aix-Marseille I

Herreman, W., Le Bars, M., \& Le Gal, P. 2009, Phys. Fluids, 21, 046602

Hollerbach, R., \& Kerswell, R. 1995, J. Fluid Mech., 298, 327

Hussmann, H., \& Spohn, T. 2004, Icarus, 171, 391

Kabin, K., Combi, M., Gombosi, T., et al. 1999, J. Geophys. Res., 104, 19

Karatekin, Ö., Van Hoolst, T., \& Tokano, T. 2008, Geophys. Res. Lett., 35, L16202

Kerswell, R. 1993a, Geophys. Astrophys. Fluid Dyn., 71, 105

Kerswell, R. 1993b, Geophys. Astrophys. Fluid Dyn., 72, 107

Kerswell, R. 1994, J. Fluid Mech., 274, 219

Kerswell, R. 2002, Ann. Rev. Fluid Mech., 34, 83

Kerswell, R., \& Malkus, W. 1998, Geophys. Res. Lett., 25, 603

Kudlick, M. 1966, Ph.D. Thesis, Massachusetts Institute of Technology

Kuskov, O., \& Kronrod, V. 2005, Icarus, 177, 550

Lacaze, L., Le Gal, P., \& Le Dizès, S. 2004, J. Fluid Mech., 505, 1

Lacaze, L., Le Gal, P., \& Le Dizès, S. 2005, Phys. Earth Planet. Int., 151, 194

Lacaze, L., Herreman, W., Bars, M., Dizès, S., \& Gal, P. 2006, Geophys. Astrophys. Fluid Dyn., 100, 299

Lavorel, G., \& Le Bars, M. 2010, Phys. Fluids, 22, 114101

Le Bars, M., \& Le Dizès, S. 2006, J. Fluid Mech., 563, 189

Le Bars, M., Le Dizès, S., \& Le Gal, P. 2007, J. Fluid Mech., 585, 323

Le Bars, M., Lacaze, L., Le Dizès, S., Le Gal, P., \& Rieutord, M. 2010, Phys. Earth Planet. Int., 178, 48

Le Dizès, S. 2000, Phys. Fluids, 12, 2762

Lebovitz, N., \& Zweibel, E. 2004, ApJ, 609, 301

Léger, A., Grasset, O., Fegley, B., et al. 2011, Icarus, 213, 1

Lifschitz, A., \& Hameiri, E. 1991, Phys. Fluids A, 3, 2644

Lissauer, J. 1985, J. Geophys. Res., 90, 11289

Lorenzani, S., \& Tilgner, A. 2001, J. Fluid Mech., 447, 111

Lorenzani, S., \& Tilgner, A. 2003, J. Fluid Mech., 492, 363

Malkus, W. 1989, Geophys. Astrophys. Fluid Dyn., 48, 123

Manglik, A., Wicht, J., \& Christensen, U. 2010, Earth Planet. Sci. Lett., 289, 619

Melosh, H. 1975, Earth Planet. Sci. Lett., 26, 353

Miyazaki, T., \& Fukumoto, Y. 1992, Phys. Fluids A, 4, 2515

Mizerski, K., \& Bajer, K. 2009, J. Fluid Mech., 632, 401
Mizerski, K., \& Bajer, K. 2011, Phys. D, 240, 1629

Morize, C., Le Bars, M., Le Gal, P., \& Tilgner, A. 2010, Phys. Rev. Lett., 104, 214501

Munk, W., \& Wunsch, C. 1998, Deep-Sea Research Part I, 45, 1977

Nettelmann, N., Fortney, J., Kramm, U., \& Redmer, R. 2011, ApJ, 733, 2

Newton, I. 1686, For an English translation, see e.g. IB Cohen and A. Whitman (Berkeley: University of California Press), in particular, see the Scholium on absolute motion therein

Noir, J., Hemmerlin, F., Wicht, J., Baca, S., \& Aurnou, J. 2009, Phys. Earth Planet. Int., 173, 141

O'Brien, D., Geissler, P., \& Greenberg, R. 2002, Icarus, 156, 152

Ogilvie, G., \& Lin, D. 2004, ApJ, 610, 477

Ogilvie, G., \& Lin, D. 2007, ApJ, 661, 1180

Ou, S., Tohline, J., \& Motl, P. 2007, ApJ, 665, 1074

Poincaré, R. 1910, Bull. Astr., 27, 321

Rieutord, M. 2000, Phys. Earth Planet. Int., 117, 63

Rieutord, M. 2003, Stellar Rotation, Proc. IAU Symp., 215, held 11-15 November, 2002 in Cancun, Yucatan, ed. A. Maeder, \& P. Eenens (San Francisco: ASP, 2004), 394

Rieutord, M., \& Valdettaro, L. 2010, J. Fluid Mech., 643, 363

Ross, M., \& Schubert, G. 1989, J. Geophys. Res., 94, 9533

Sauret, A., Cébron, D., Morize, C., et al. 2010, J. Fluid Mech., 662, 260

Sohl, F., Hussmann, H., Schwentker, B., Spohn, T., \& Lorenz, R. 2003, J. Geophys. Res, 108

Sumita, I., \& Yoshida, S. 2003, Earth's Core: Dynamics, Structure, Rotation, 213 Tilgner, A. 2007, Phys. Rev. Lett., 99, 194501

Tobie, G., Mocquet, A., \& Sotin, C. 2005, Icarus, 177, 534

Valencia, D., Ikoma, M., Guillot, T., \& Nettelmann, N. 2010, A\&A, 516, A20

Van Hoolst, T., Rambaux, N., Karatekin, Ö., \& Baland, R. 2009, Icarus, 200, 256

Van Hoolst, T., Rambaux, N., Karatekin, Ö., Dehant, V., \& Rivoldini, A. 2008, Icarus, 195, 386

Van Hoolst, T., Sohl, F., Holin, I., et al. 2007, Space Sci. Rev., 132, 203

Wagner, F. W., Sohl, F., Rückriemen, T., \& Rauer, H. 2011, IAU Symp. 276, ed. A. Sozzetti, M. G. Lattanzi, \& A. P. Boss, 193

Wahr, J., Zuber, M., Smith, D., \& Lunine, J. 2006, J. Geophys. Res., 111

Webb, D. 1982, Geophys. J. Roy. Astron. Soc., 70, 261

Wicht, J., \& Tilgner, A. 2010, Space Sci. Rev., 152, 501

Wieczorek, M., \& Le Feuvre, M. 2009, Icarus, 200, 358

Williams, G. 2000, Rev. Geophys., 38, 37

Williams, G. 2004, The Precambrian Earth: Tempos and Events, Developments in Precambrian Geology, 12, 448

Williams, J., Boggs, D., Yoder, C., Ratcliff, J., \& Dickey, J. 2001, J. Geophys. Res., 106, 27933

Winn, J., Matthews, J., Dawson, R., et al. 2011, ApJ, 737, L18

Zhang, K., Chan, K., \& Liao, X. 2011, J. Fluid Mech., 673, 468

Zimmer, C., Khurana, K., \& Kivelson, M. 2000, Icarus, 147, 329 